ORO- $5197-10$

STUDY OF HYDROCARBON - SHALE INTERACTION

PROGRESS REPORT 非10

PART IV

APPENDICES

Appendix B (continued)

Appendix C

Appendix D

Appendix E

WOTICE MN ONLI

PORTIONS OF THIS REPORT ARE IILEGIBLE. It oopy to permit the broadest best avallablo ability.

\title{
Juniata Co1lege
}

Huntingdon, Pennsylvania 16652

\section{MASTER}




\section{DISCLAIMER}

This report was prepared as an account of work sponsored by an agency of the United States Government. Neither the United States Government nor any agency Thereof, nor any of their employees, makes any warranty, express or implied, or assumes any legal liability or responsibility for the accuracy, completeness, or usefulness of any information, apparatus, product, or process disclosed, or represents that its use would not infringe privately owned rights. Reference herein to any specific commercial product, process, or service by trade name, trademark, manufacturer, or otherwise does not necessarily constitute or imply its endorsement, recommendation, or favoring by the United States Government or any agency thereof. The views and opinions of authors expressed herein do not necessarily state or reflect those of the United States Government or any agency thereof. 


\section{DISCLAIMER}

Portions of this document may be illegible in electronic image products. Images are produced from the best available original document. 
axy 3 
Sample ID : WU\#5-EGSP: 3037

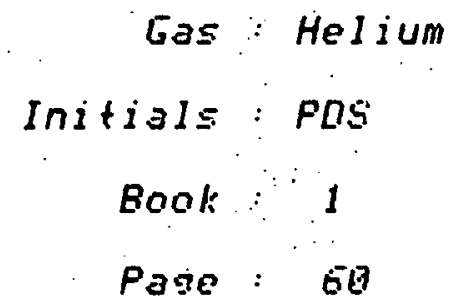

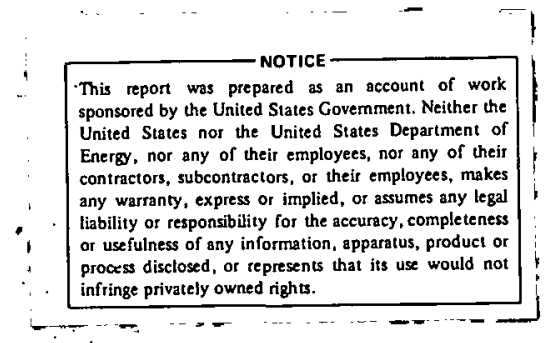

SAMPLE DITTH

Weisht : 4.9754 (aroms)

Density : 2.4288 (amec)

Sample Temr: a cent?

Particle size: . 085 (cm) $\frac{F}{3}$

DATA COLLECTER

SLOPE OF ISOTHERM

4. $91279 E-65$

2. $54961 E-093$

3. $73372 E-02$

9. $02421 E-11$

Wal GasNol Rock Torr

llal Gas.llal rockPSI

Ual Gasul RackitM

Moles Gas Gram Rack Torr

UNCERTIINTY : $3.82259: \%$

DETAILED DATA A POINTS TIKEN

PRESSURE

Torr

0

242.819

538.544

829.678

894.225
ADS GAS

Moles/Gram

a $\therefore$ 百

1. $74129 E-67$

4. $32305 E-197$

8. $62791 E-B P$

8. $54268 E-197$
UNCERTIINTY

$\%$

\section{0 .}

15. 7176

8. 90969

5. 92548

6.436
FLAE

a $\therefore$

$1 \quad \ldots$

1

1

$1 \quad \therefore \theta$

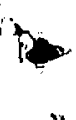

TOTICE WHN ONTX

PORTIONS OF TUIS REPORT ARE ILLEGIBLE. IQ has been reprocuc e from trie vest available copy to permit the broadestpossible aval. ability. 


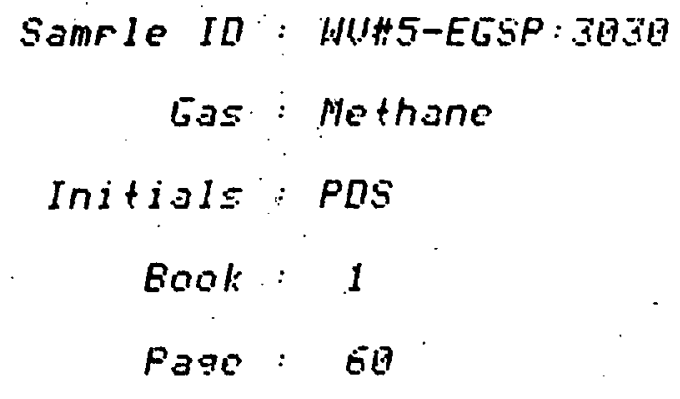

SAMPLE DATTH

$$
\begin{aligned}
& \text { Weisht : } 4.9754 \text { (aroms? } \\
& \text { Density : 2.4288 (ampe) } \\
& \text { Somrle Temr: } 9 \text { (cont) } \\
& \text { Particle size: As5 (cm) } \\
& \text { DATA COLLECTEO }
\end{aligned}
$$$$
\text { SLOPE DF ISOTHERN }
$$$$
\text { 3. 11485E-64 Ual GasNol Rack Tor }
$$$$
\text { 1.55913E-BZ Uol Gasollol rackPSI }
$$$$
229128 \text { Vol Gaswol fock }
$$$$
\text { 5.53792E-la Moles Gas Gram Rock Tor }
$$$$
\text { UNCERTIINTY : .626743 } \%
$$$$
\text { DETAILEU DATA A PUINTS TAKEN }
$$

PRESSURE Torr

(a) 239.592 526.439 821.483 913.735

\section{ADS GNS}

Molesuran:

UNCERTAINTY

$\%$

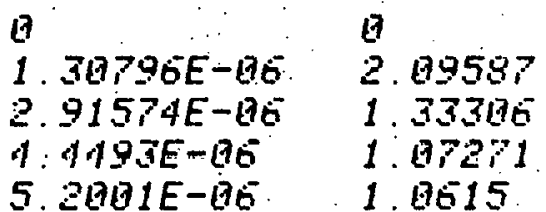

B

1. 3BT.9E-

1 : $1993 E-90^{\circ}$

5. $2801 E-80$
FLAE

6
1
1
2
1
FLAE Parameter

a

(a)

(a)

82.331 


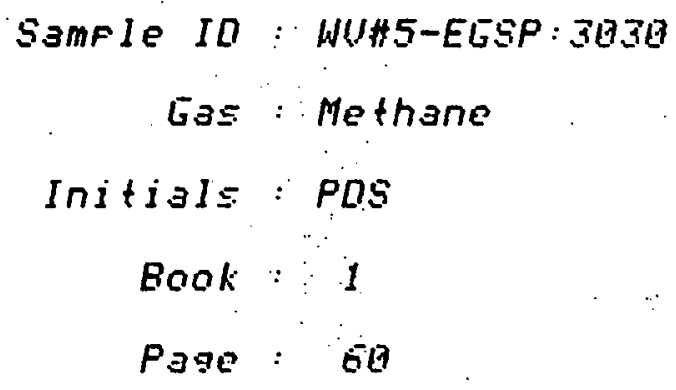




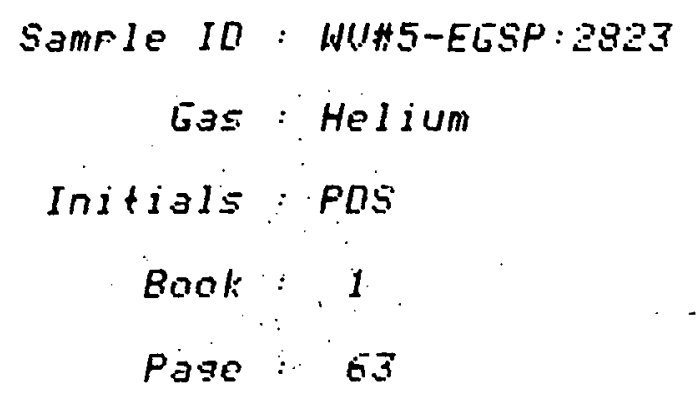

SAMPLE DITA

$$
\begin{aligned}
& \text { Weight : A. } 81121 \text { (arams? } \\
& \text { Density : 2.PBdA (amec) } \\
& \text { Samrle Temr: } a \text { (cent) } \\
& \text { Particle sizo: . } 095 \text { (cm? } \\
& \text { DPTA COLLECTED }
\end{aligned}
$$$$
\text { SLOPE. OF ISOTHERM }
$$$$
\text { 8.37848E-85 Wal Gas Ual Rocklotor }
$$$$
\text { 4. 32879E-193 Uol Gas Ual rack.PSI }
$$

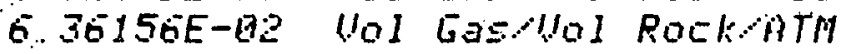$$
\text { 1. 38B87E-G9 Moles Gas Gram Rock Torr }
$$

$$
\text { UNLERTIINTY : 2.64428\% }
$$

DETAILED DNTA A POINTS TAKEN

PRESSURE

Torr

$\theta$

235.187

524.231

815.949

887.261
ADS EAS

Moles.trim.

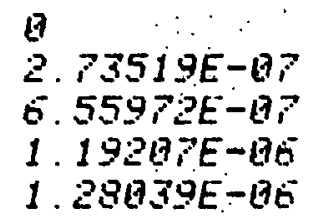

UNCERTIINTY

$$
\therefore
$$

$\dot{F} L A E$

B

19. 1975

6. 1.1701

4. 15818

4.40587
FLAE

Parameter

(3)

B

B

B 


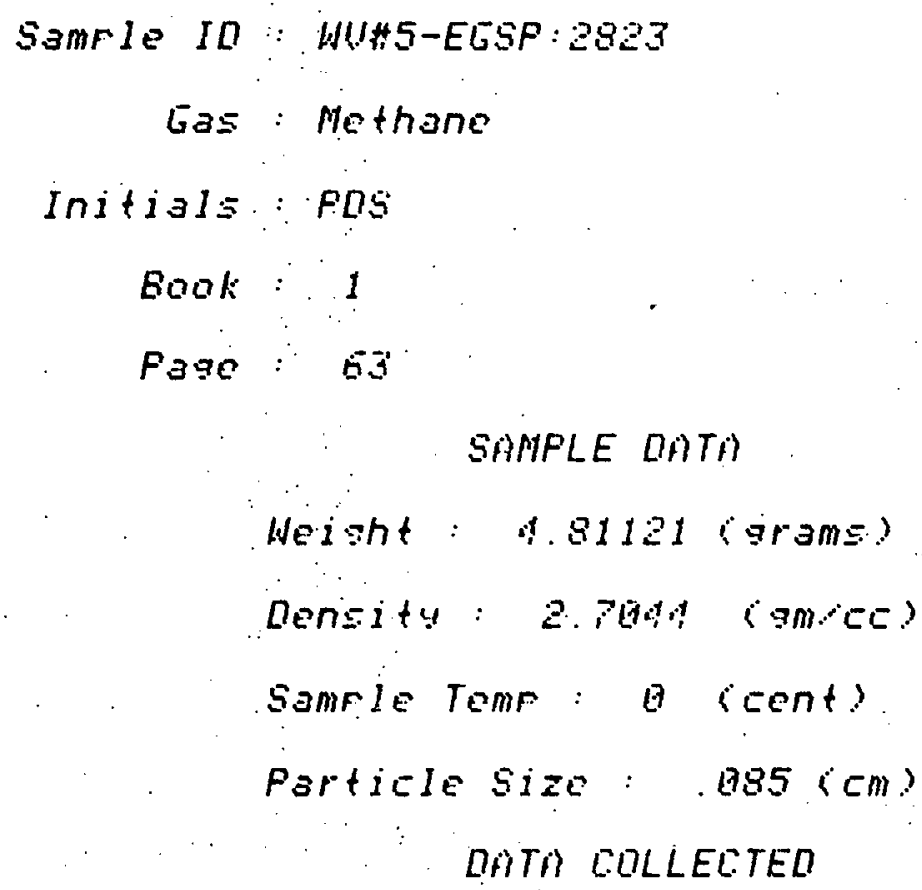

SL UPE OF ISOTHERM

3. 26943E-6\% Ual Gas Vol Rock Torr. 1 65975E-62 Ual Gas vol rock PSI 243910 UOl Gasvol RackATH

5.29455E-69 Moles Gas Gram Rock Tor

UNCERTAINTY : . B99201 $\%$

DETAILED DATA A POINTS TAKEN

PRESSUIRE

Torr

B

232.981

514.957

808.969

879.982
ADS GAS:

Molesuram

1. $25755 E-06$

2. $69246 E-196$

4. $319401 E-60$

$4.6478 E-196$
UNCERTAINTY $\because$

9
2.26729
1.59141
1.15929
1.23498
FLAG

10
FLAG
Parameter

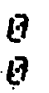

B

日 


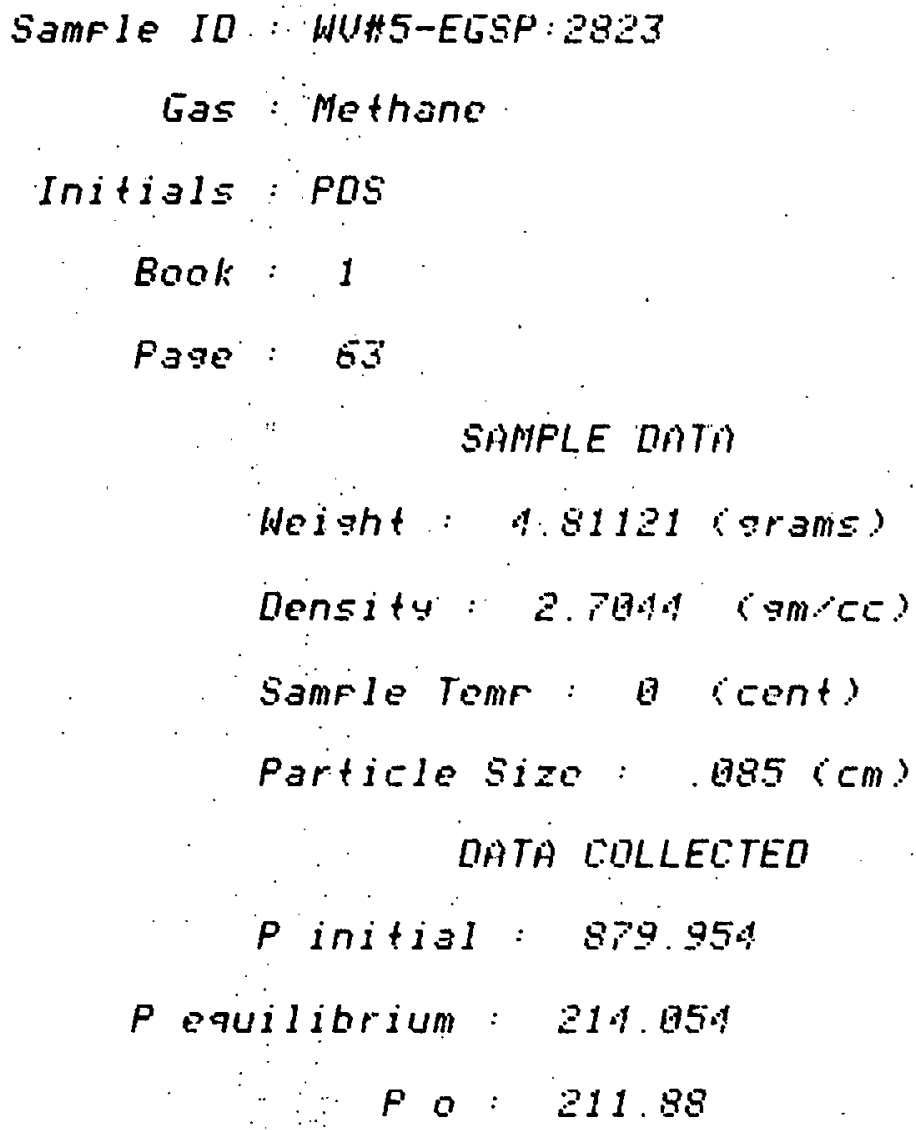




$$
\begin{aligned}
& \text { Sample ID : HU\#5-EGSF:2823 } \\
& \text { Gas : Nitrosen } \\
& \text { Initisls : POS } \\
& \text { Book } 1 \\
& \text { Page } \therefore \text { : } 3 \\
& \text { SAMPLE DITH } \\
& \text { Weisht : M. B1121 iorams? } \\
& \text { Density : 2. Plad (amer) } \\
& \text { Samrle Temp: } \theta \text { (cent) } \\
& \text { Particle size: } 085(\mathrm{~cm}) \\
& \text { DATA COLLEETEO }
\end{aligned}
$$

INTERHAL AREA

$$
\begin{aligned}
& \text { 9. } 41991 E-195 \\
& 3.48318 E-135 \\
& 5.76045 \\
& 8.96385
\end{aligned}
$$

Males:

Molesoml

ml. $905 \mathrm{ml}$. rock

specific Area isq. metersoram?

$$
\text { UNCERTAINTY : . } 185487 \%
$$

C. : $\quad 319.3543$

UNCERTAINTY IN $C=.188920 \%$

BAROMETRIL PRESSURE $739.939 \mathrm{~mm} \mathrm{HO}$

$$
\text { DETAILED DATA S PUINTS TAKEN }
$$

PRESSURE

Torr

B

3. 80231

73.6195

136.625

$188.730^{\circ}$

240.753
ADS GAS

Molesuram.

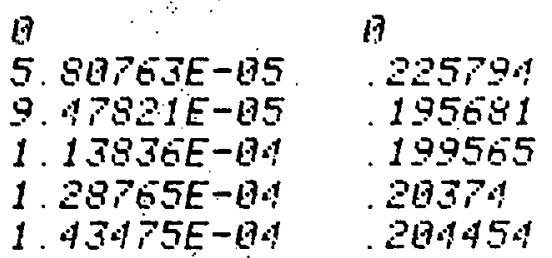

FLAE

FLAE

UNCERTAINTY

$\because$

Parameter 


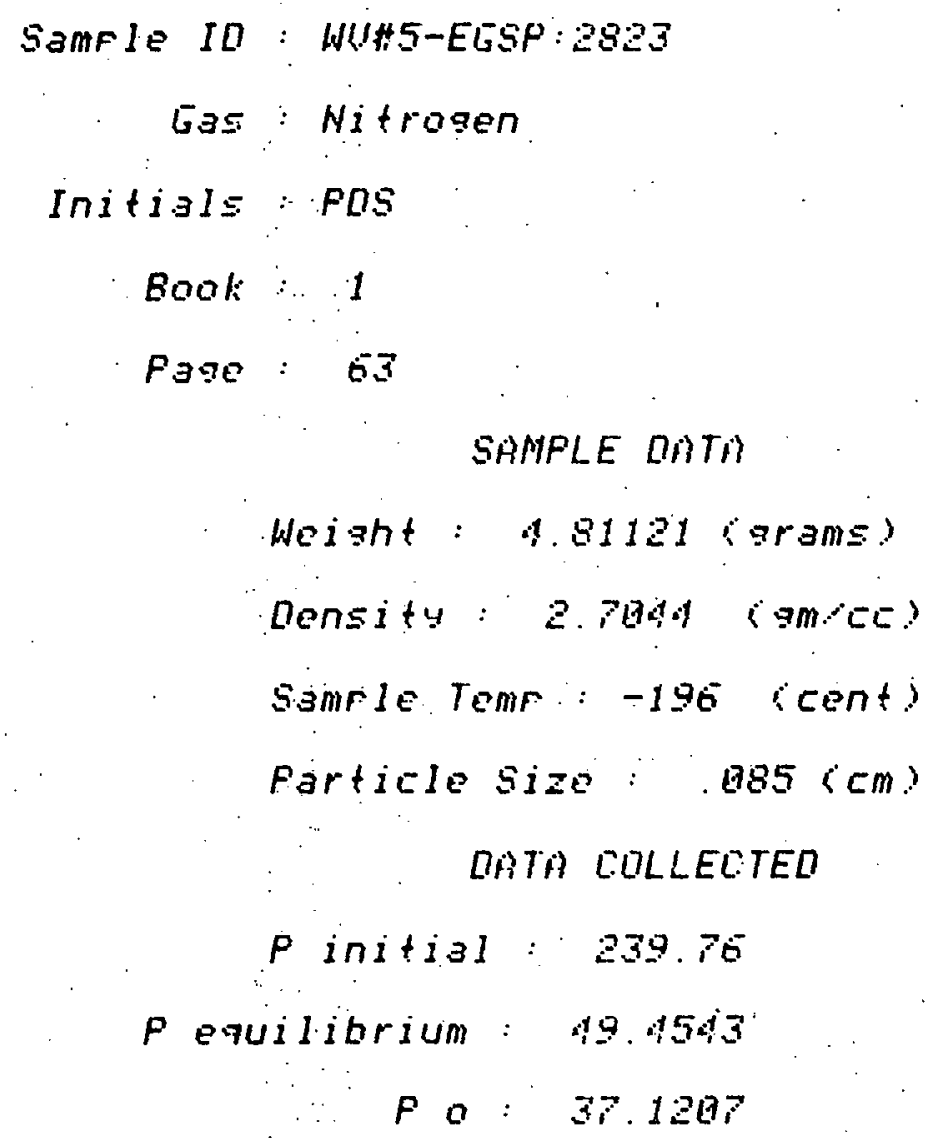




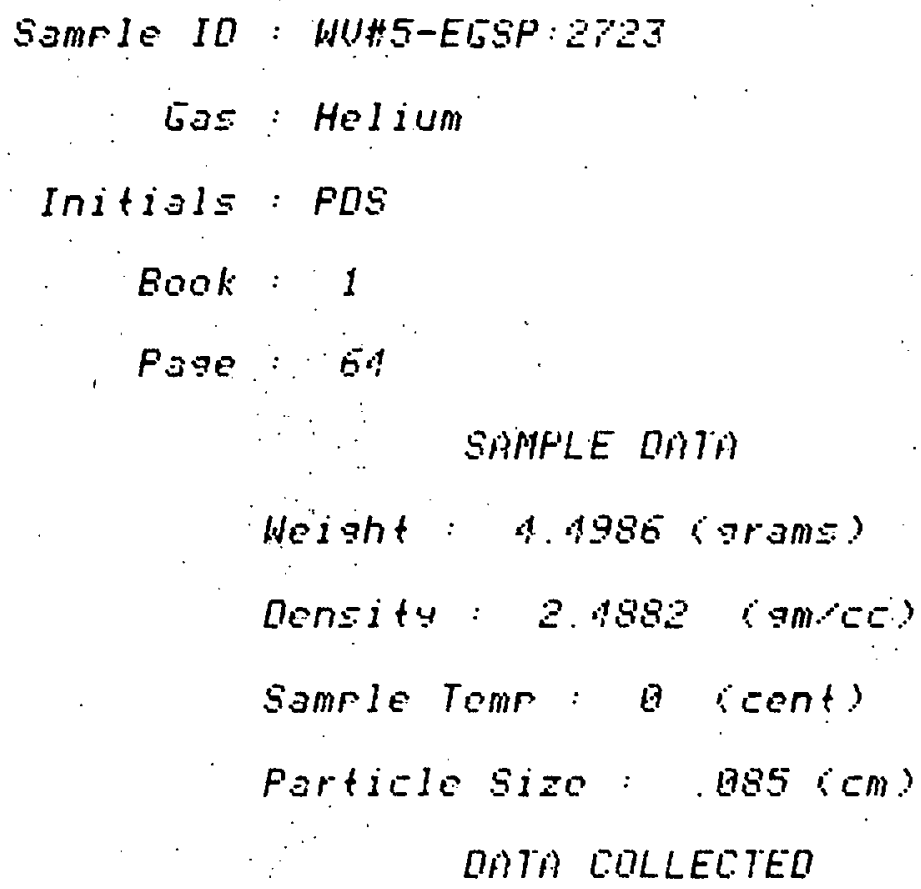

SLOFE OF ISOTHERM

3. $827280-85$

1. $97928 E-63$

2. $98873 E-82$

Wal GasNol Rack Torr

Wal GavNol rock psi

$6.80243 E-10$

Ual Gasulval Fock hth

Moles Gasuram Rock Tar

UNCERTIINTY : $5.04789 \%$

FRESSUIRE

Torr

B

236.827

531.053

820. 044

888.814

DETAILEO DATA a POINTS TOKEN

NDS GNS

Molesurom

\begin{abstract}
(6)
\end{abstract}
1. 19462EE-G7

2. $89055 E-B 7$

o. $20091 E-B \vec{O}$

6. POEQ9E-BP
LINCERTAINTY

$\therefore$

$\theta$

25. 1527

1.1: 9

8. 51799

‥ 02681
FLAE

FLAE

Parameter 


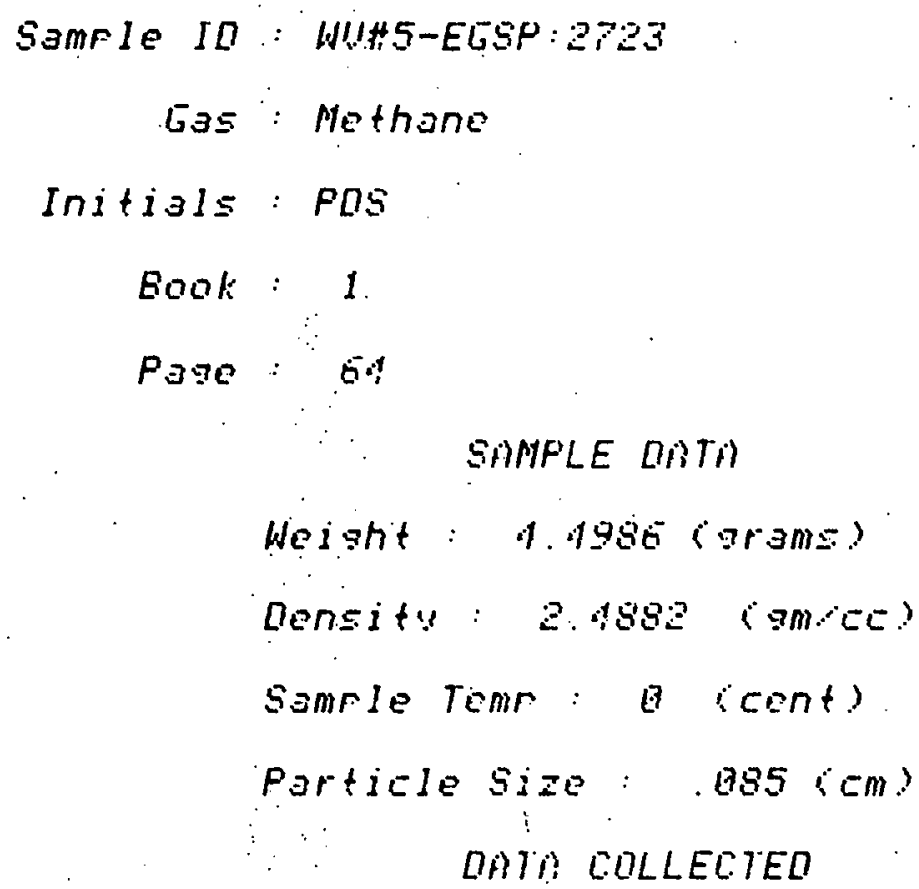

SLOFE OF ISOTHHERH

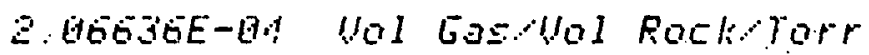

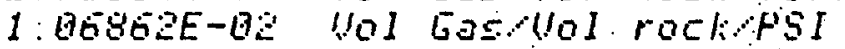

15704. Vol Gasullol Rackill

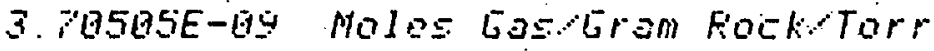

LINLEFTHINTY: $1.00245:$

DETHILEQ DITH A POINTS THKEN

PRESSURE

Torr

6

234.94

518.594

812.268

889.521
ADS Lits

Molegroam

B

7. $84676 E-B 7$

1. $83119 E-136$

3. $86992 E-196$

3. $38775 E-105$
UNCERTAINTY

$*$

FLAG

17

3. 87927

2. 35695

1. 72604

1. 84898
FLAG

Parametar

日

$B$

3

8 


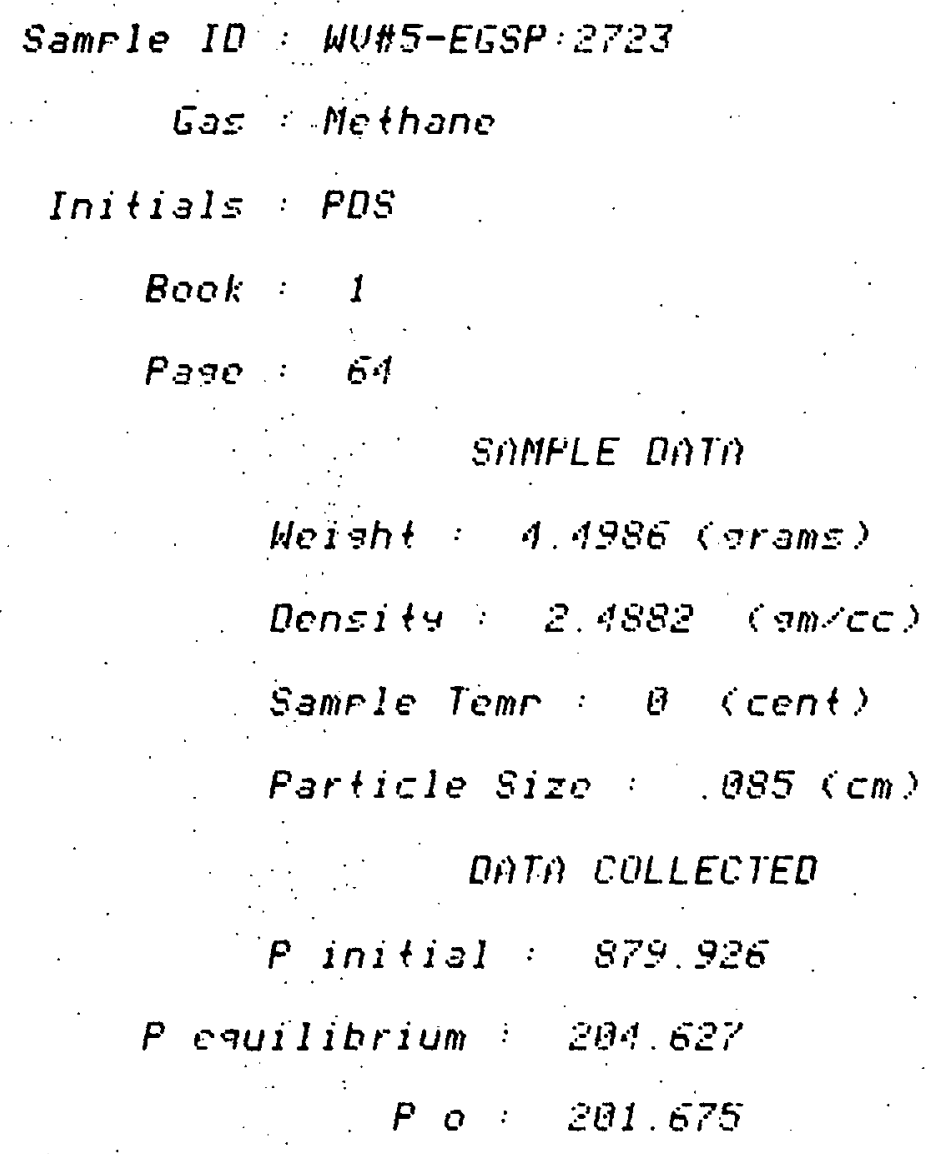

SPECIFIC DELASIBILTY 5.9A7BE-BS UNCERTATNTY . BSB324\%

Sustem of Moles of $2.03 B 7 E-B 6$ 

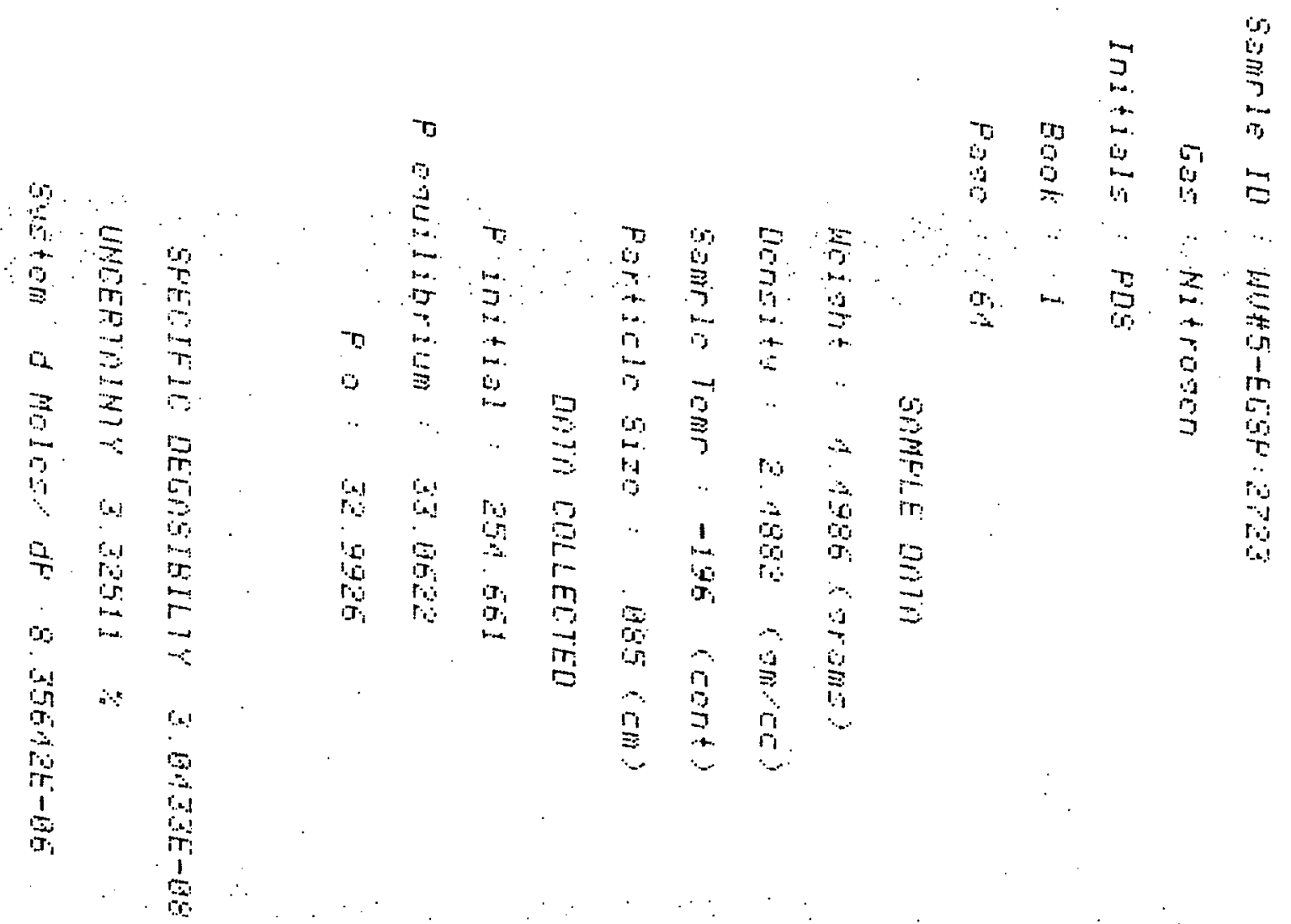


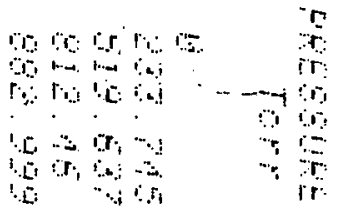

r.

$r a r a b a$

ar a

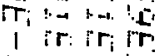

$=1 \frac{1}{1}$

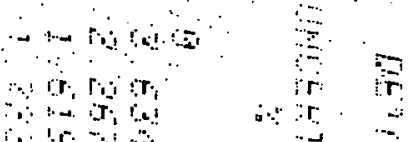

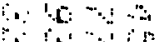

ir

n............... is

8

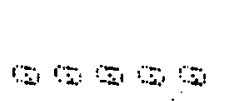

$\begin{array}{ll}0 & = \\ 0 r & = \\ 0 & =\end{array}$

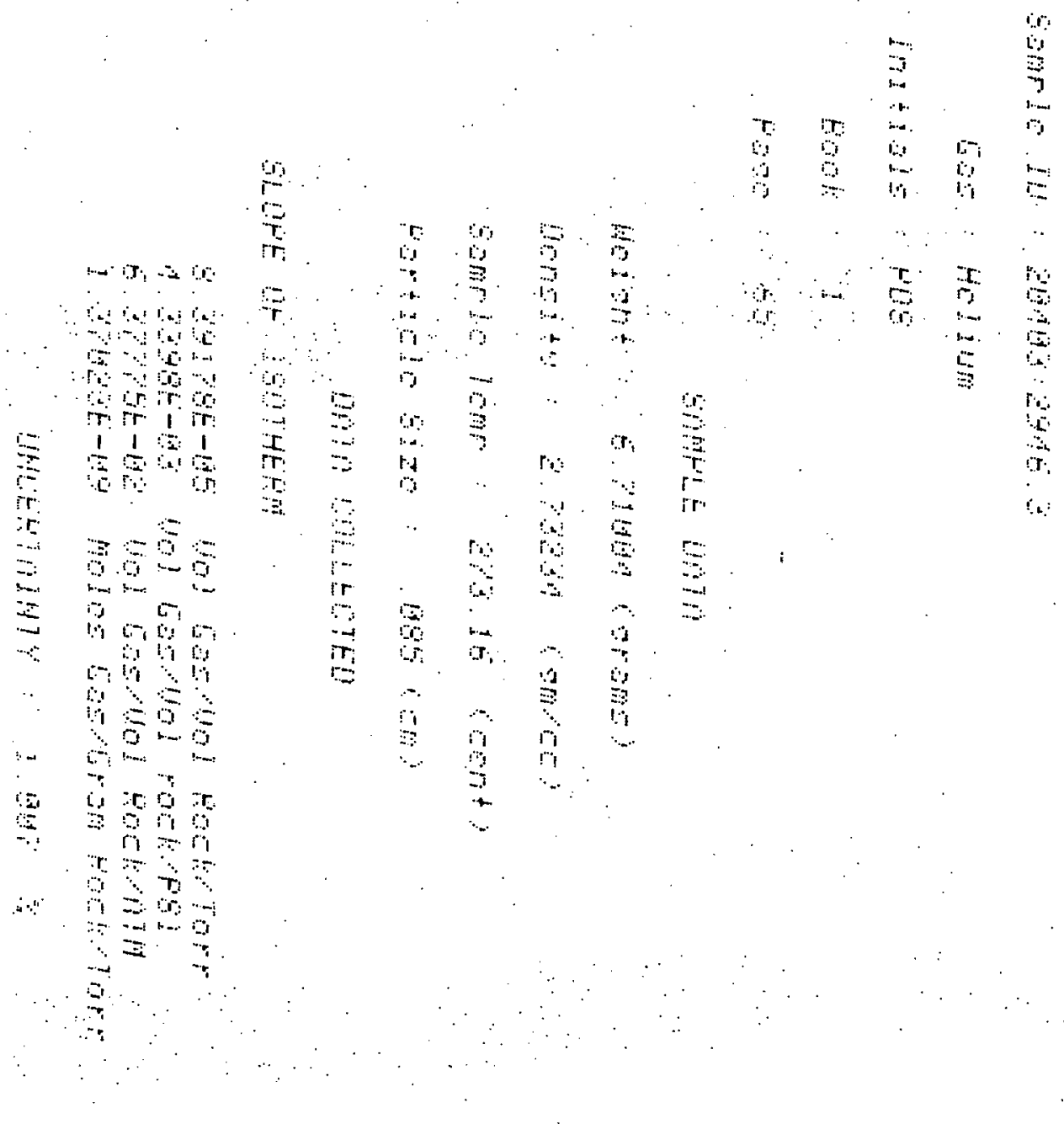




\begin{tabular}{|c|c|}
\hline $\begin{array}{lll}0 & 0 \\
0 & 0 \\
0 & 0\end{array}$ & $\begin{array}{r}r \\
r r \\
-r\end{array}$ \\
\hline 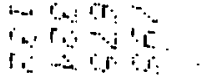 & \\
\hline
\end{tabular}

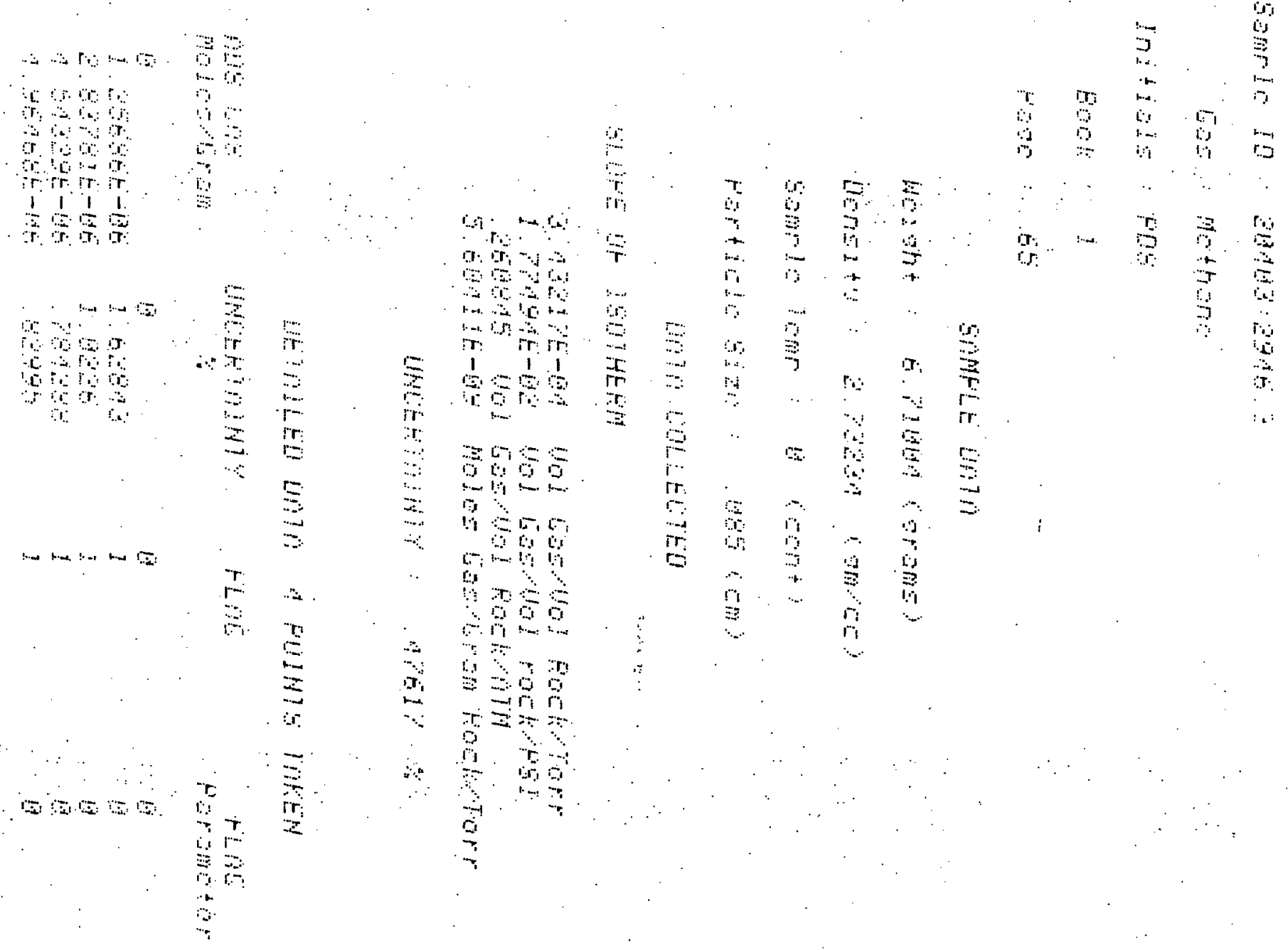




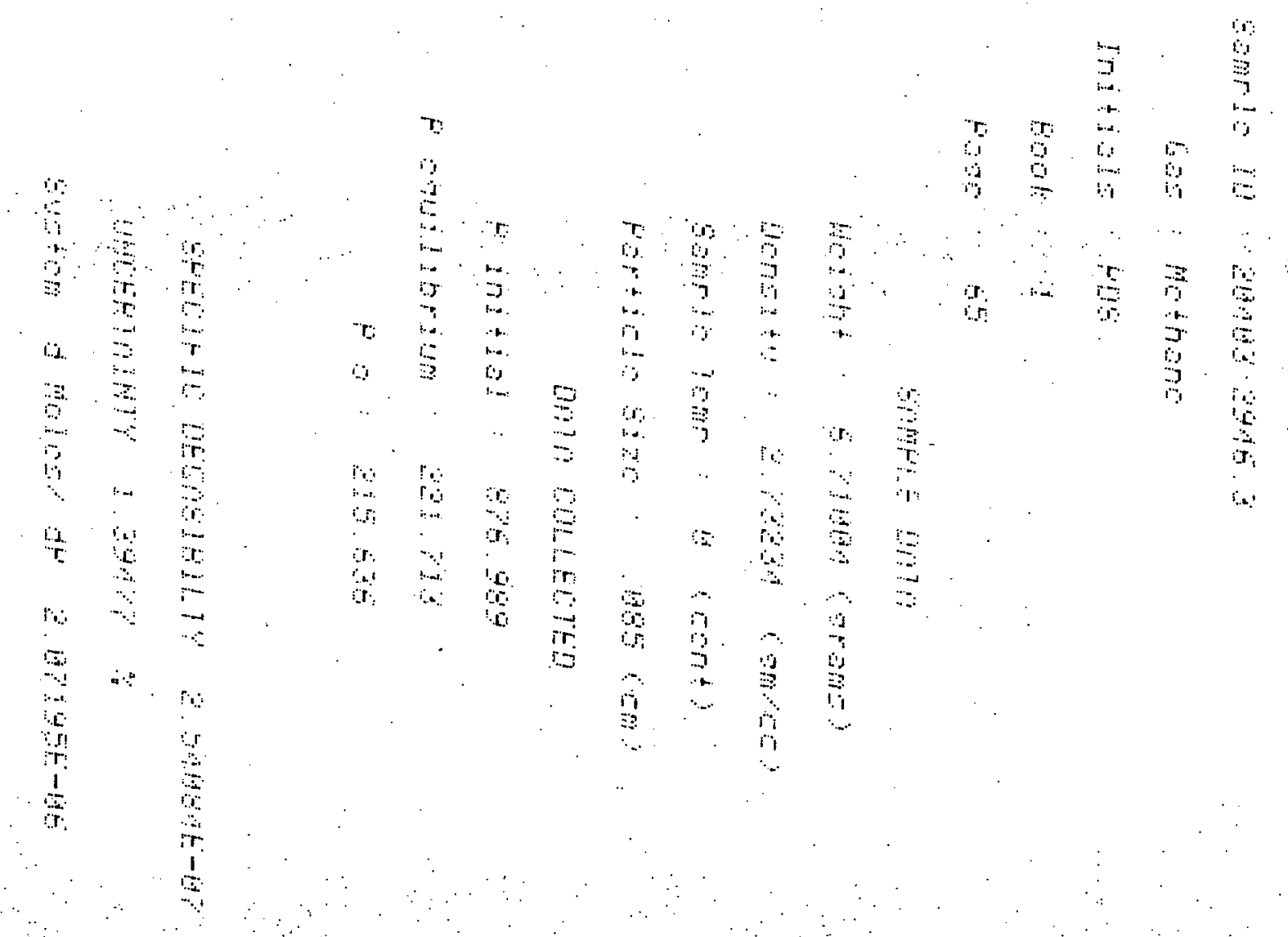




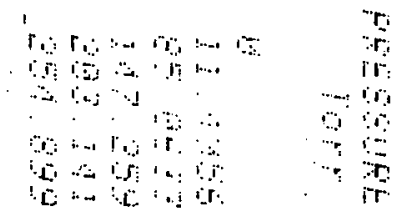

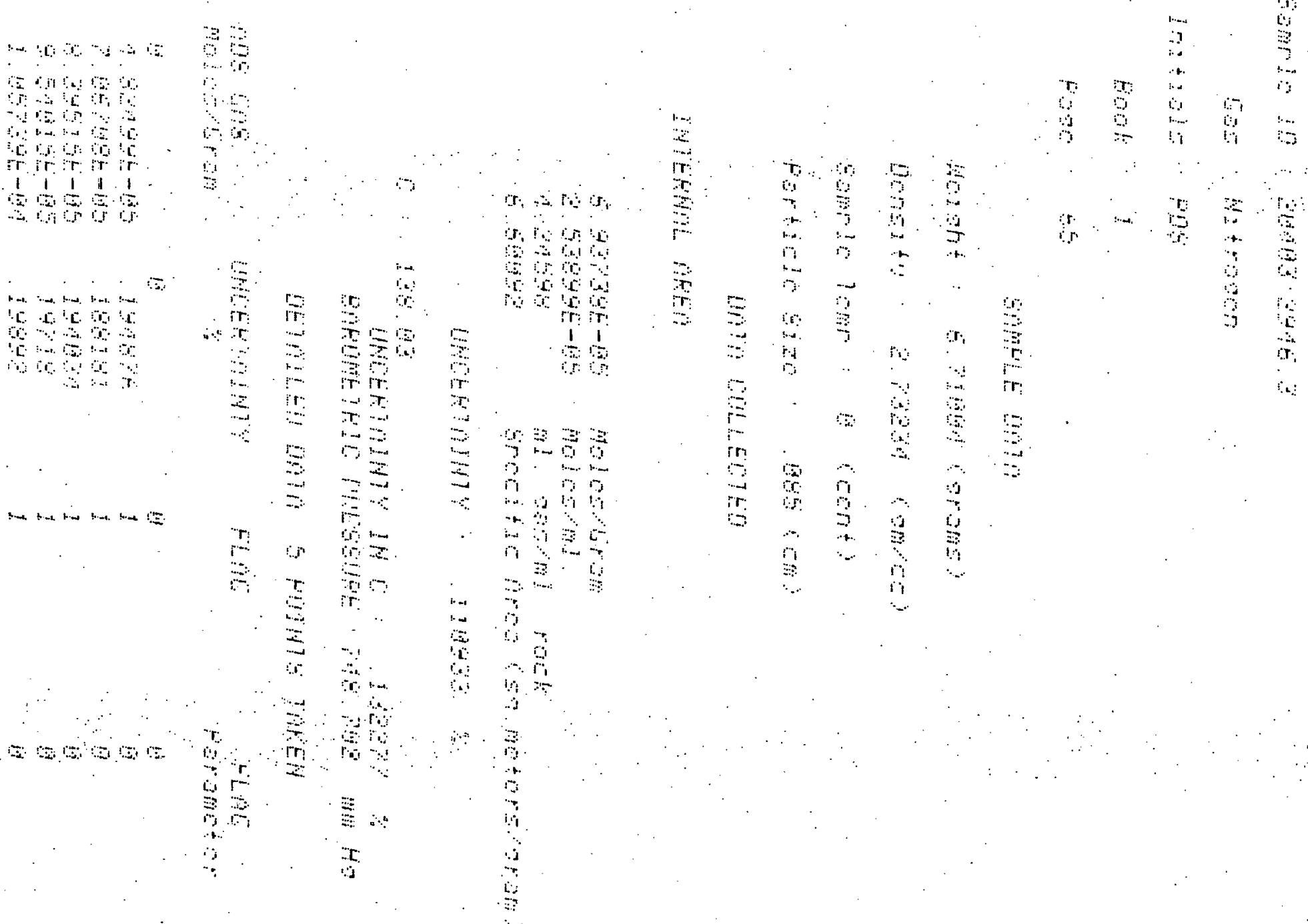




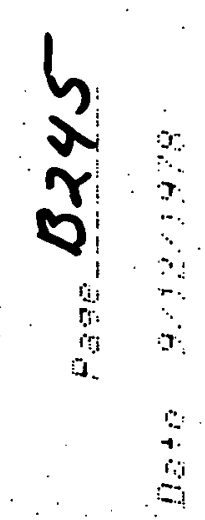

3
3
0
0
0
0

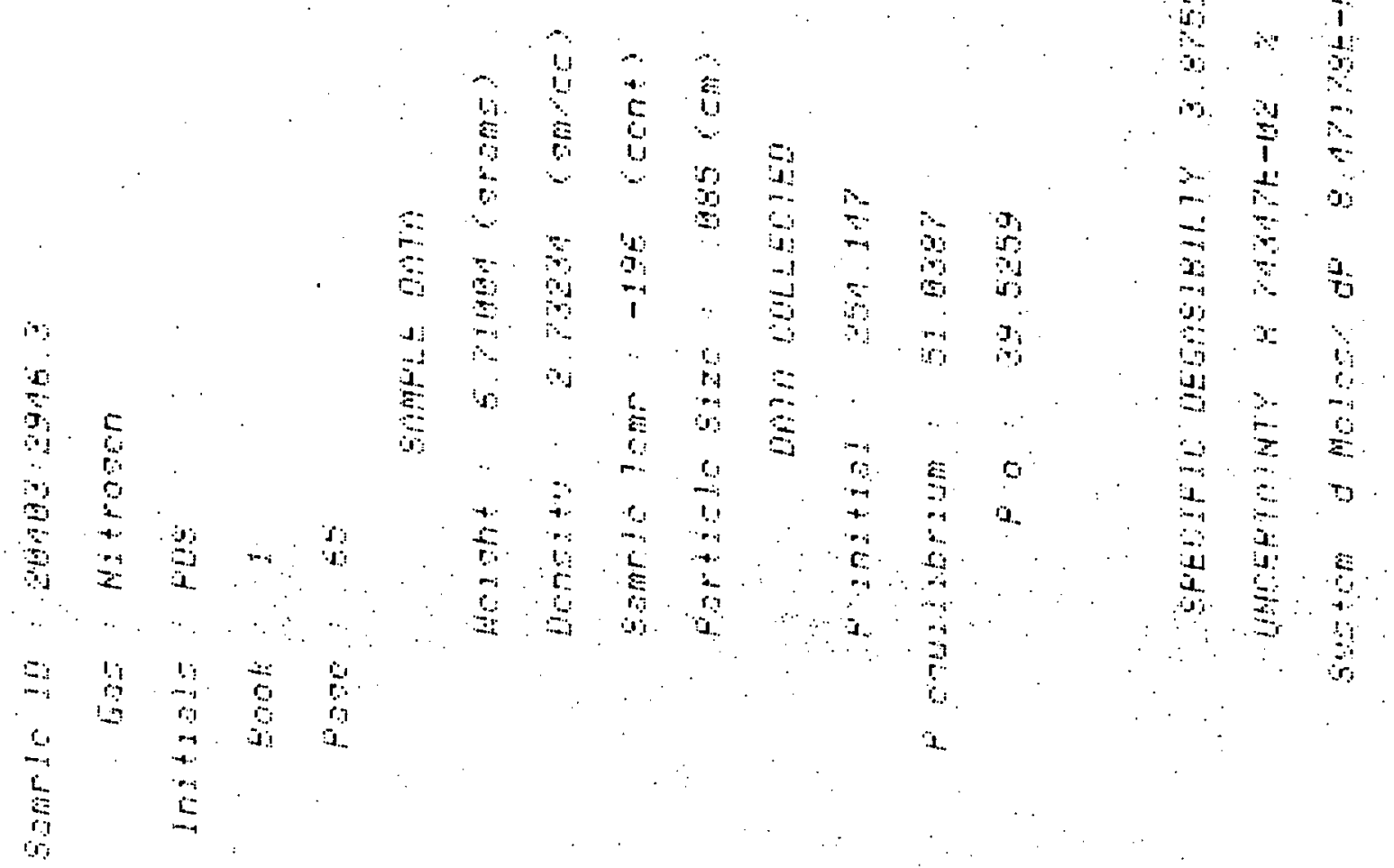


a. $x_{i}+40 \%$

1
$y-a$

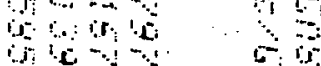

at 0

,

$\frac{1}{2} \frac{1}{2}$

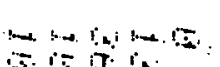

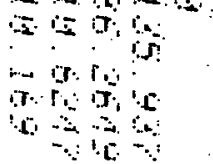

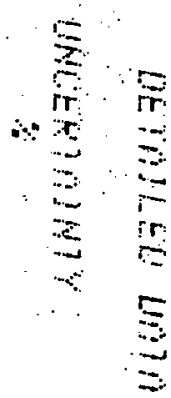

$$
5
$$

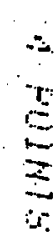

$\operatorname{sos} 20$

$\frac{2}{2}$

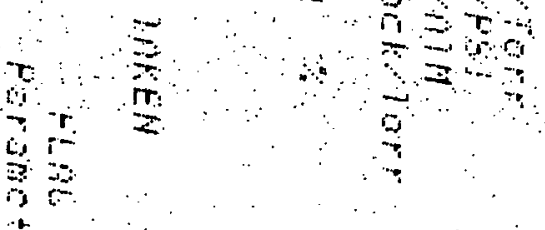




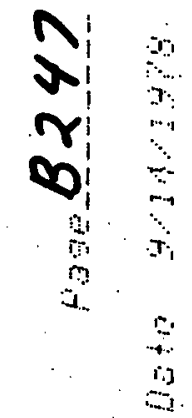

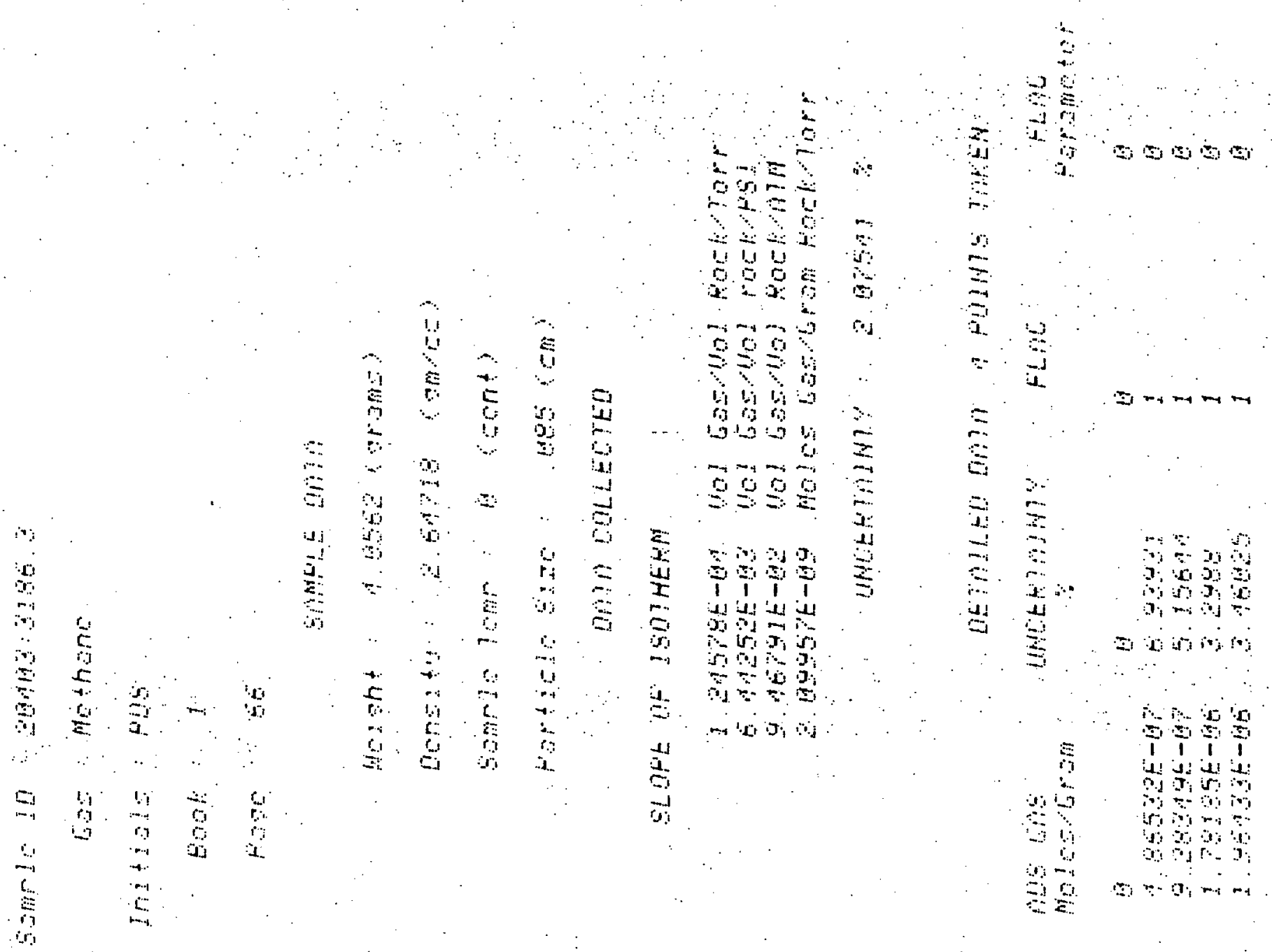

$\frac{5}{1}$ 


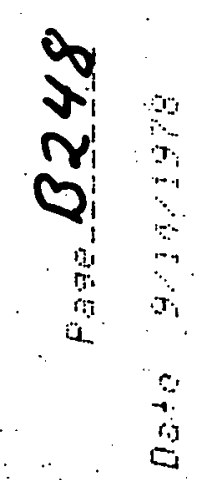

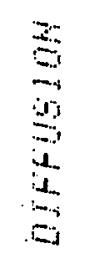

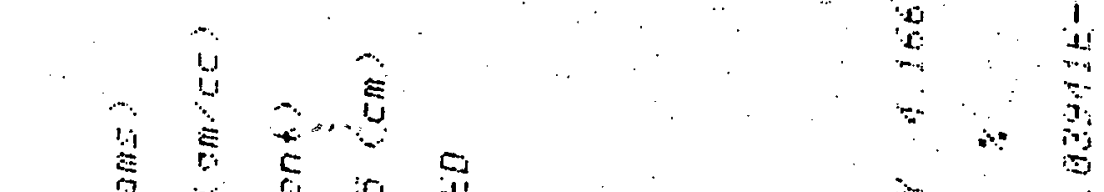

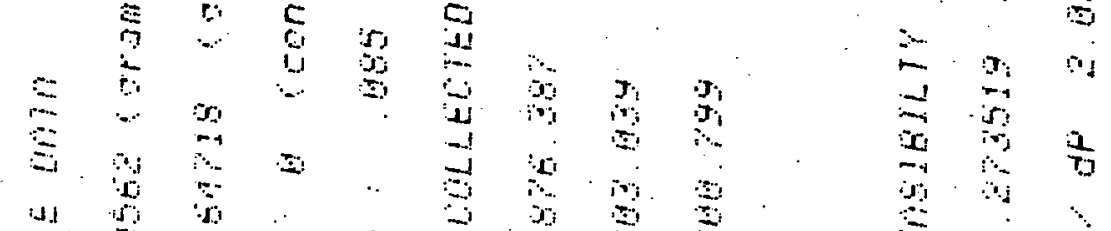

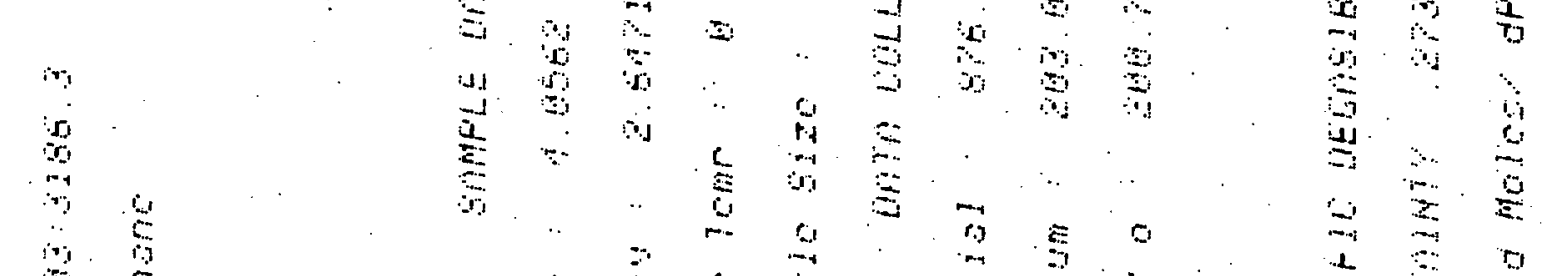

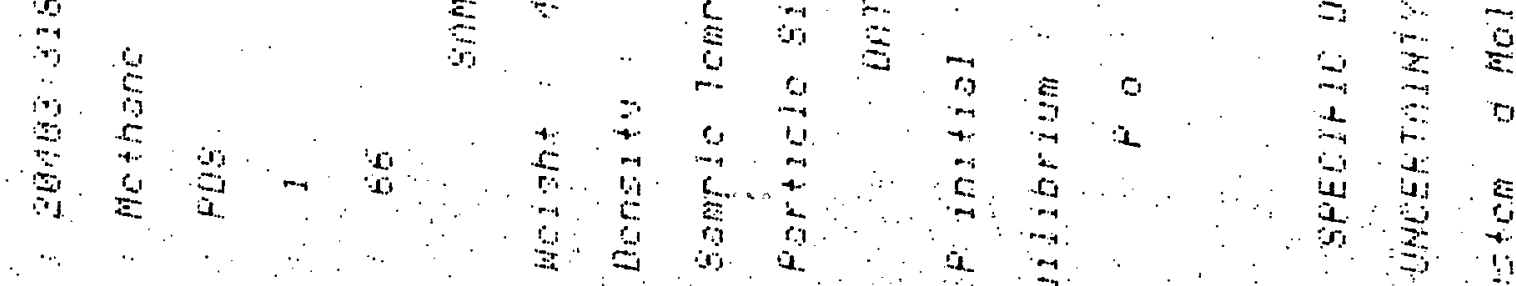

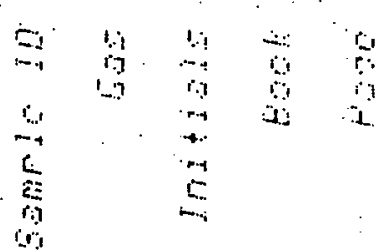

$$
\begin{aligned}
& \text { a } \\
& \frac{u}{4} \frac{4}{5} \\
& a \text {. }
\end{aligned}
$$




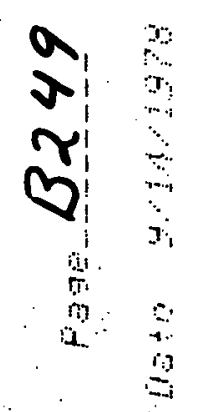

1

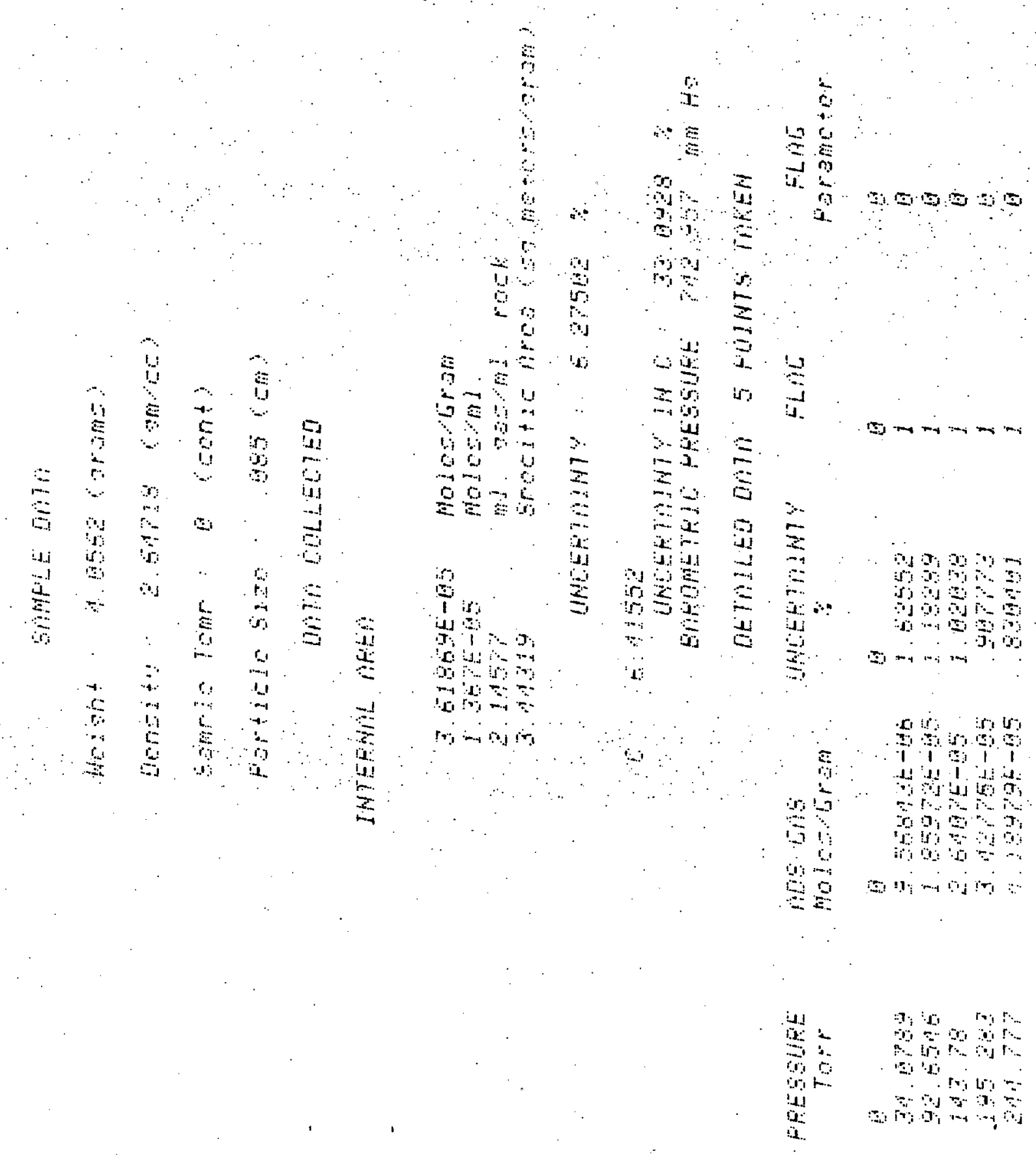


$\begin{array}{ll}0 \\ n \\ n & 0 \\ 0 & 0 \\ 0 & 0 \\ 0 & 0 \\ 0 & 0 \\ 0 & 0\end{array}$

3
3
3
1
8

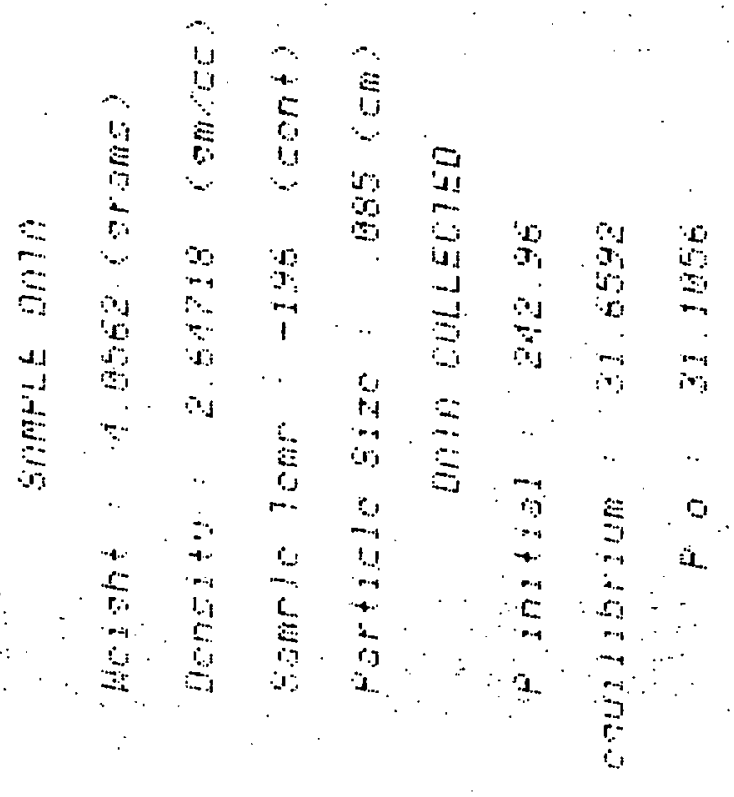

I.

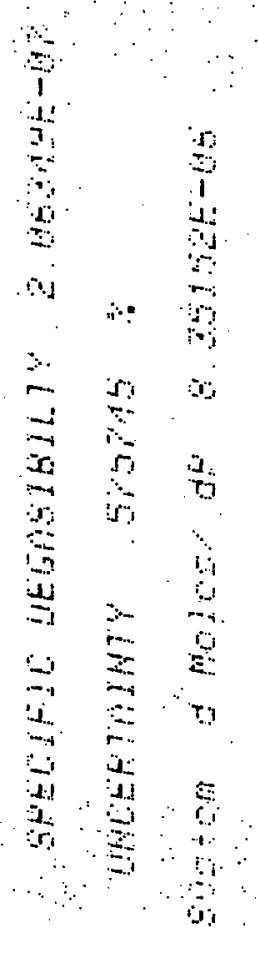




\begin{tabular}{|c|c|}
\hline $\begin{array}{ll}0 & 10 \\
0 & 0\end{array}$ & \\
\hline 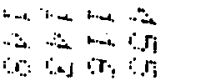 & \\
\hline
\end{tabular}

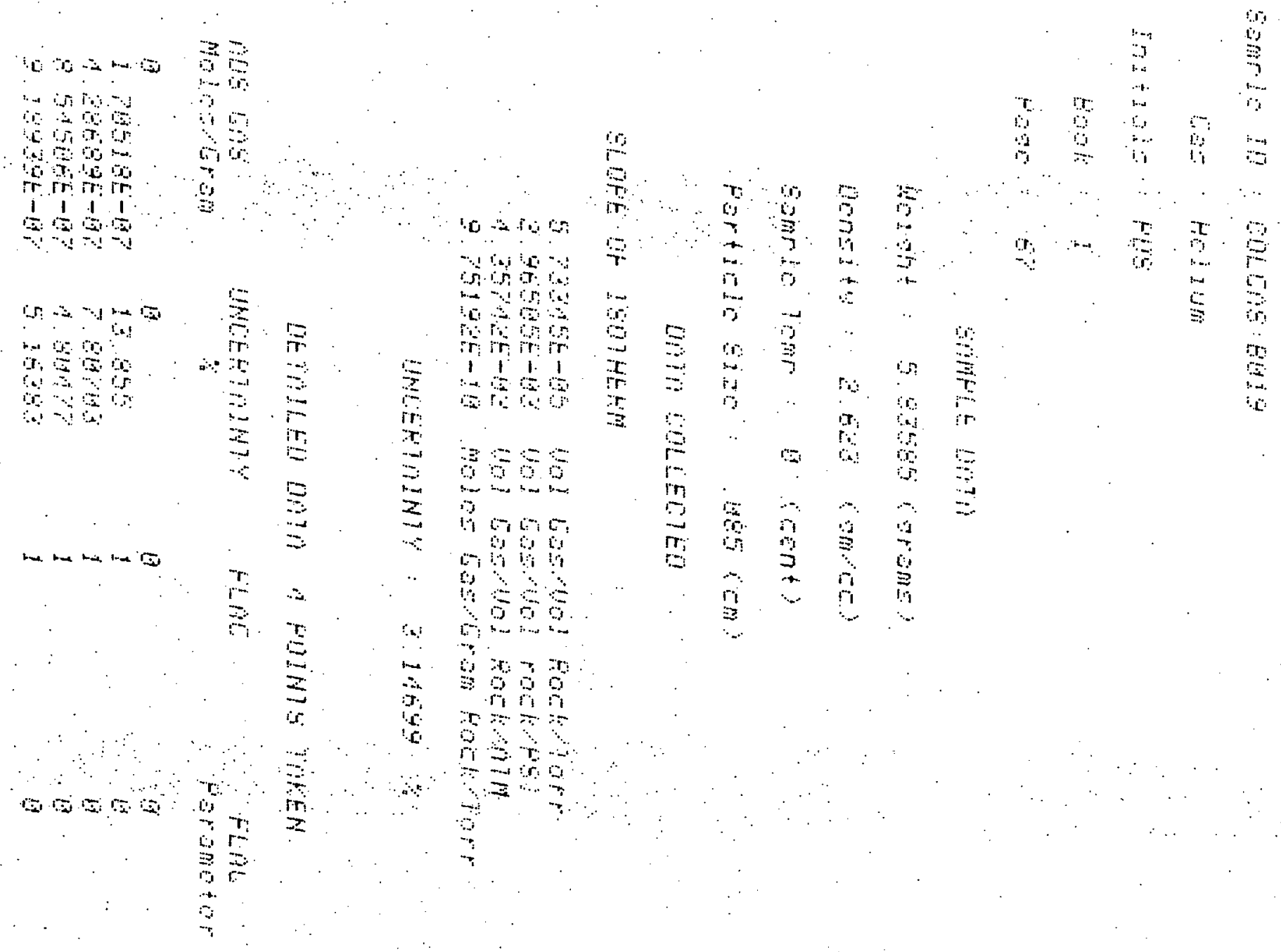


$\begin{array}{lll}r & r \\ 4 & r\end{array}$

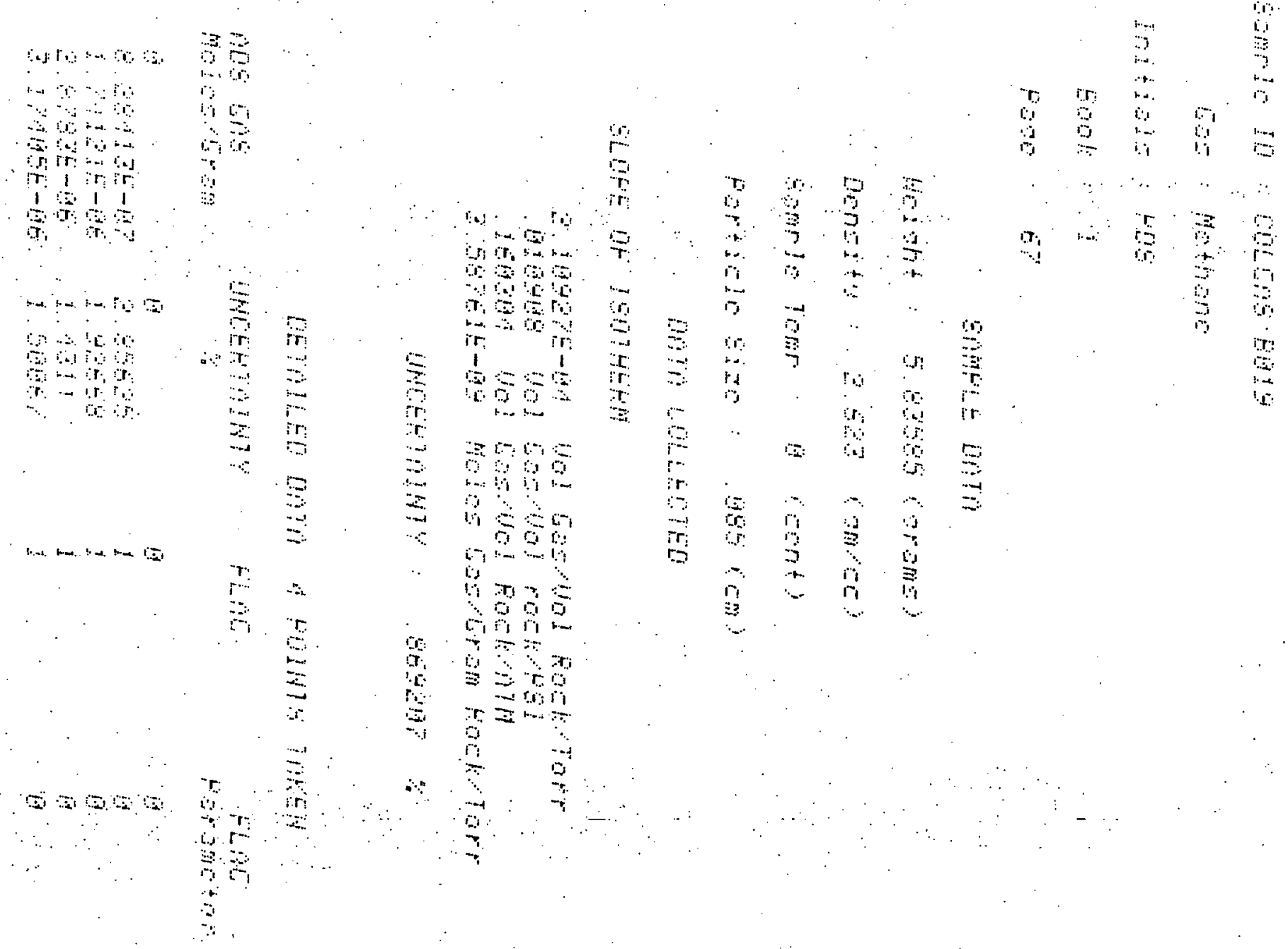


$m$
$m$
$n$
$n$
$n$
0
0
0
0
0
0
0
0

3
$\mathrm{u}$
$\mathrm{u}$
$\mathrm{u}$

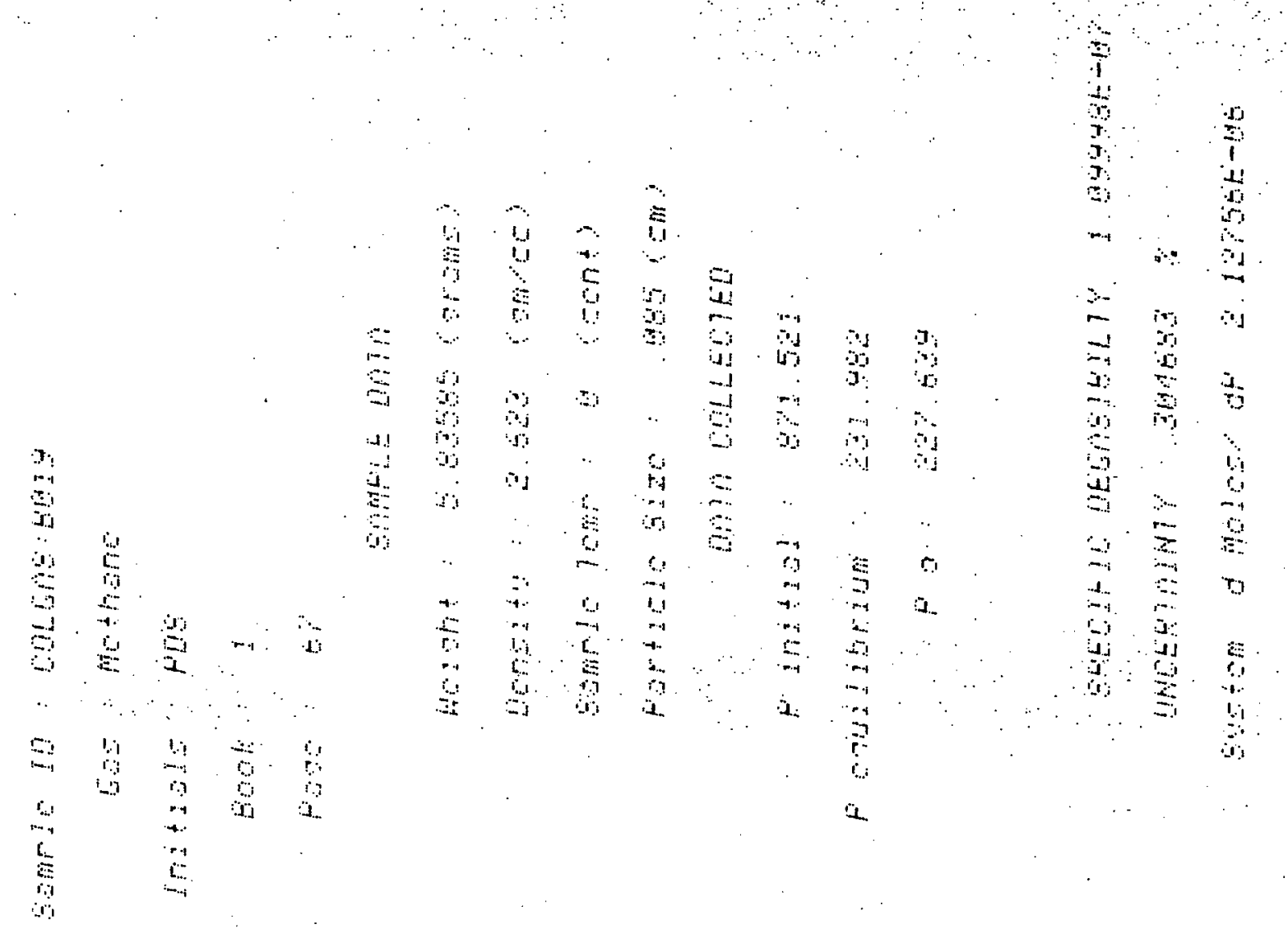




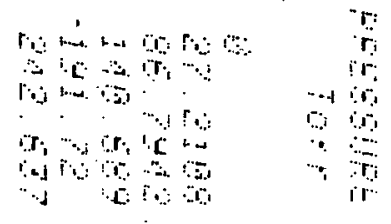

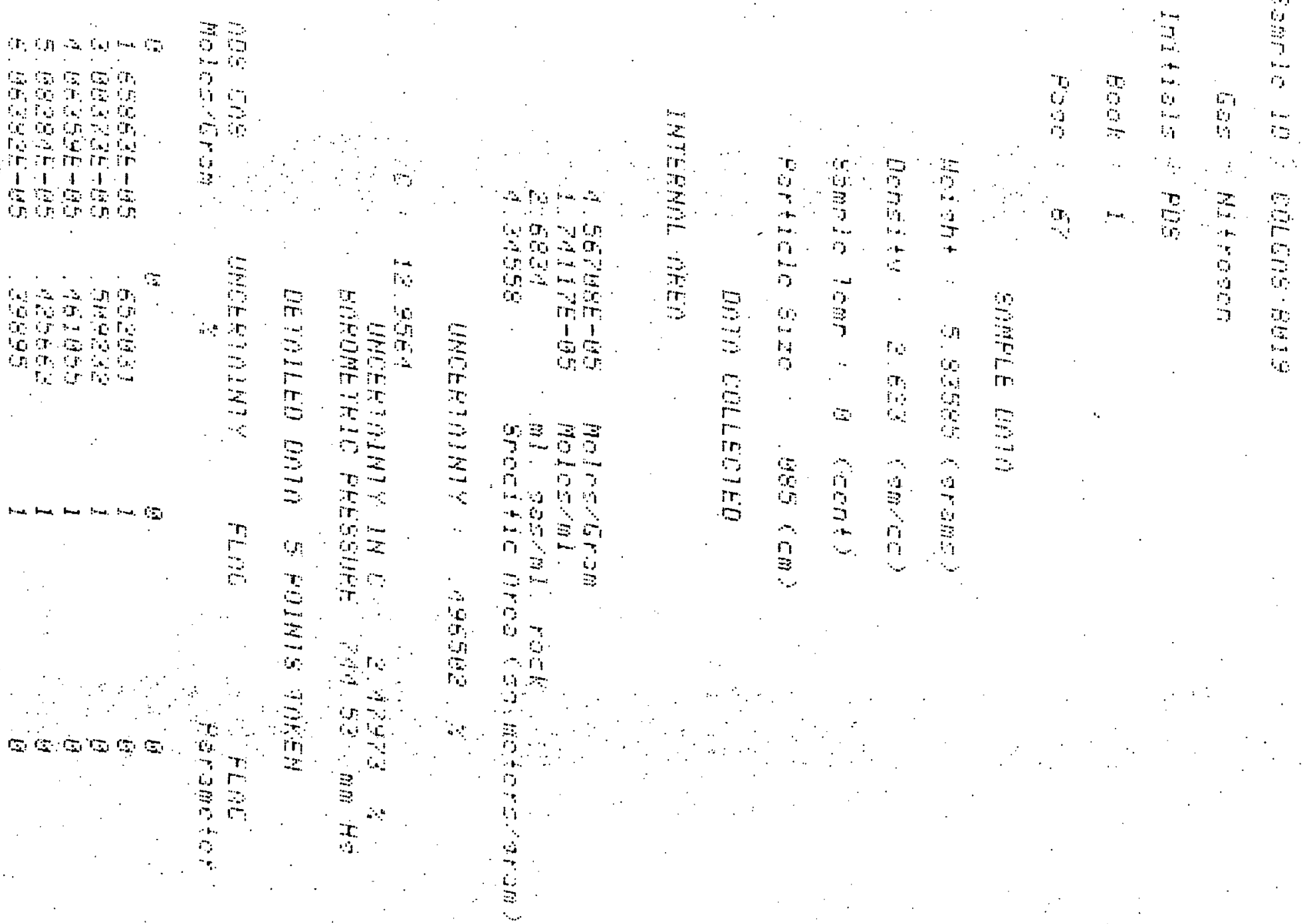




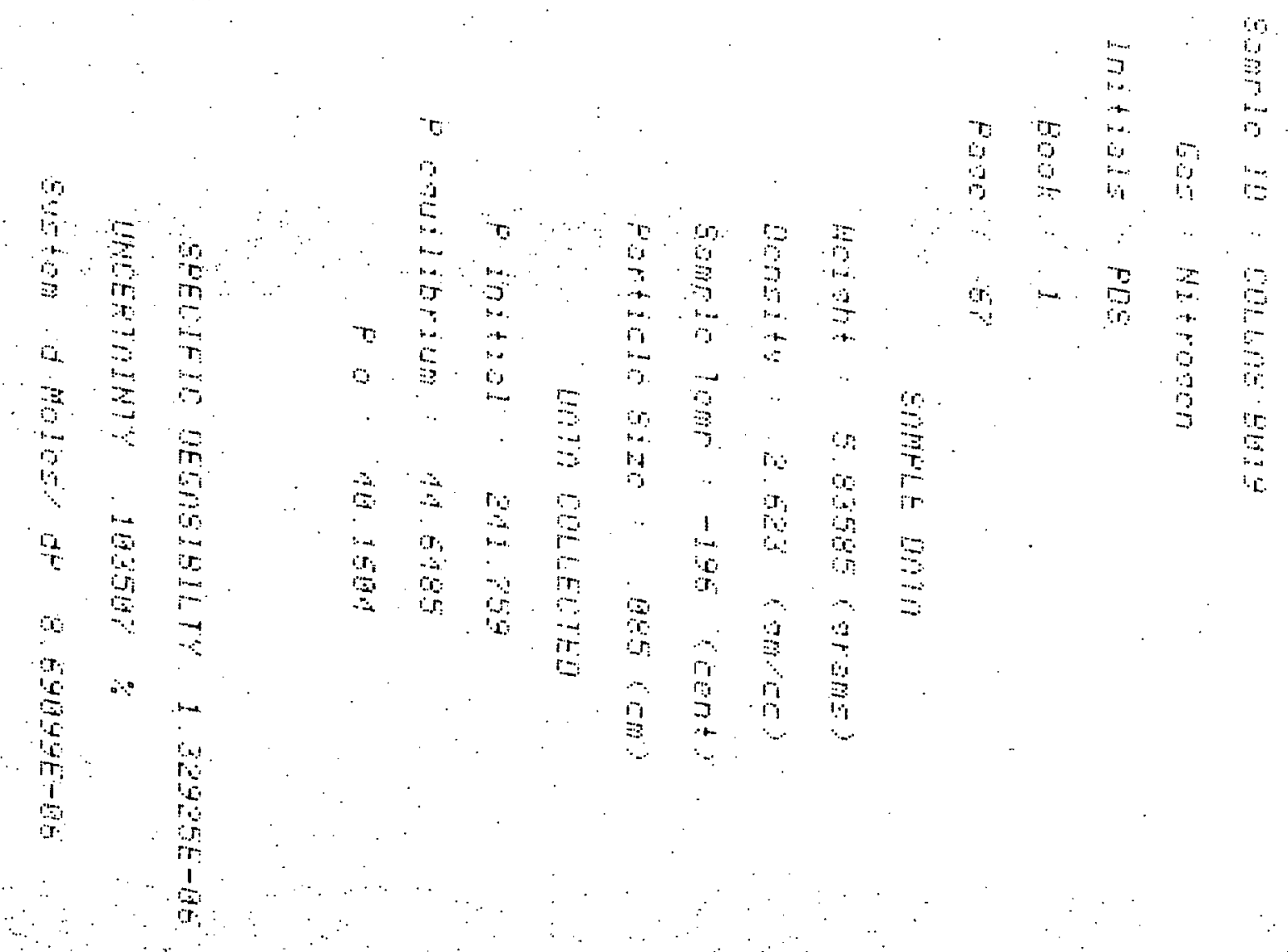




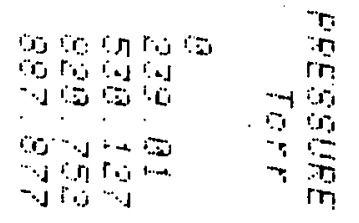

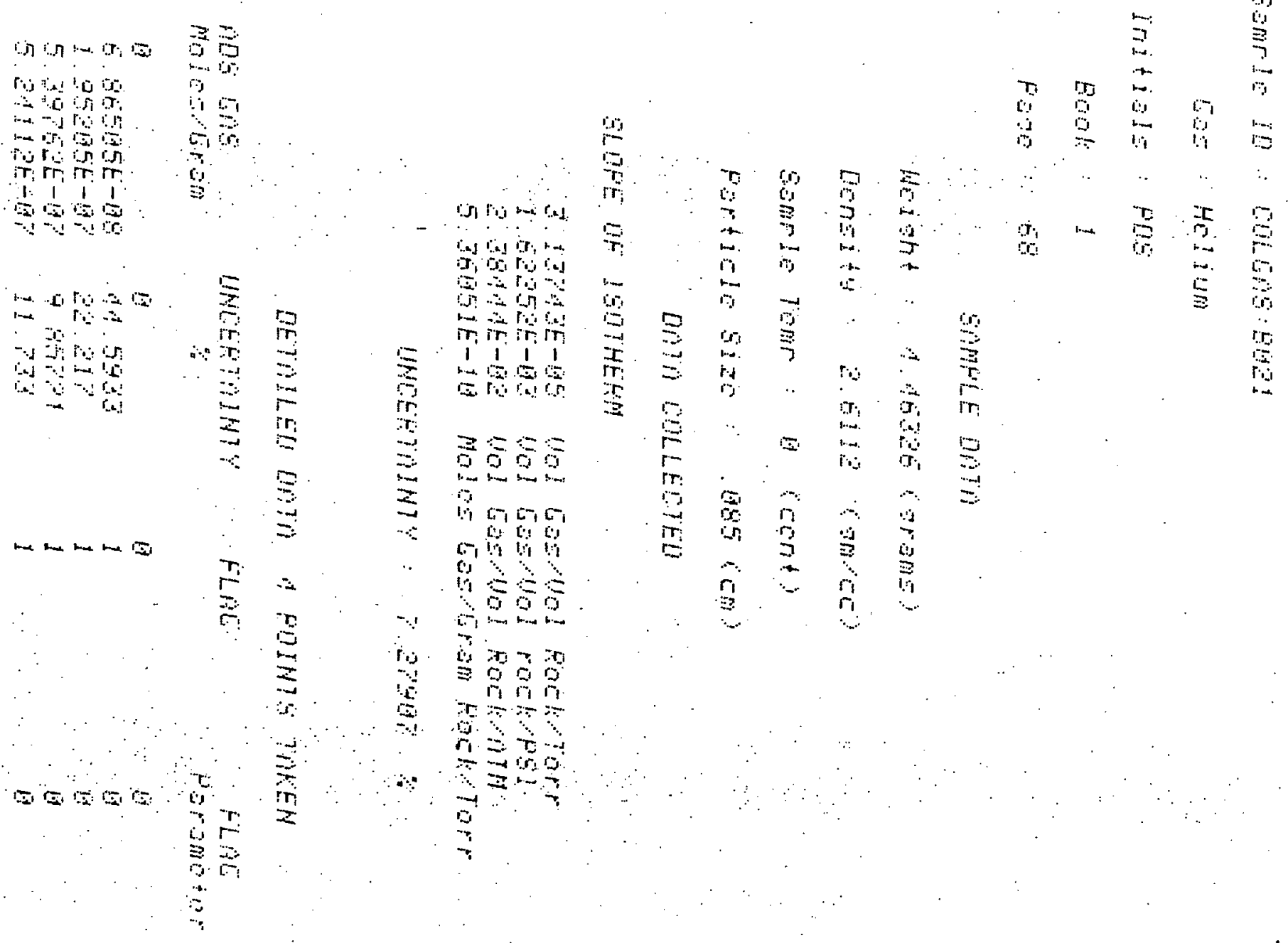




\begin{tabular}{|c|c|}
\hline 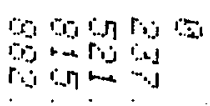 & $\begin{array}{r}r \\
-1 \\
-10\end{array}$ \\
\hline 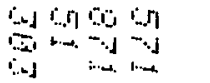 & \\
\hline
\end{tabular}

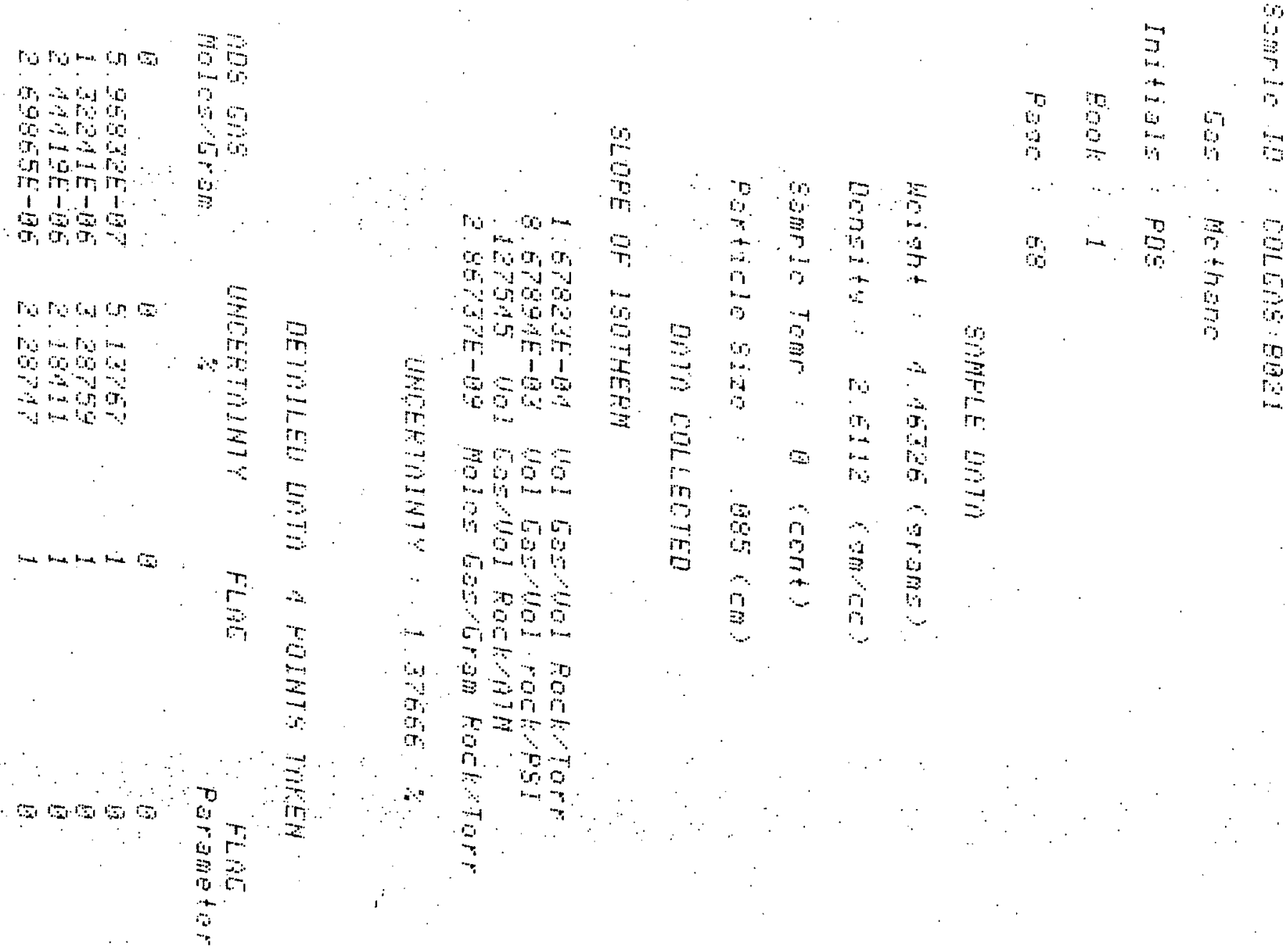


$\begin{array}{lll}\infty & \\ b & 0 \\ \gamma & 0 \\ 0 & \vdots \\ 0 & 0 \\ 0 & 0 \\ 0 & 0 \\ 0 & 0 \\ 0 & 0\end{array}$

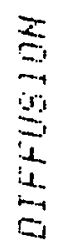

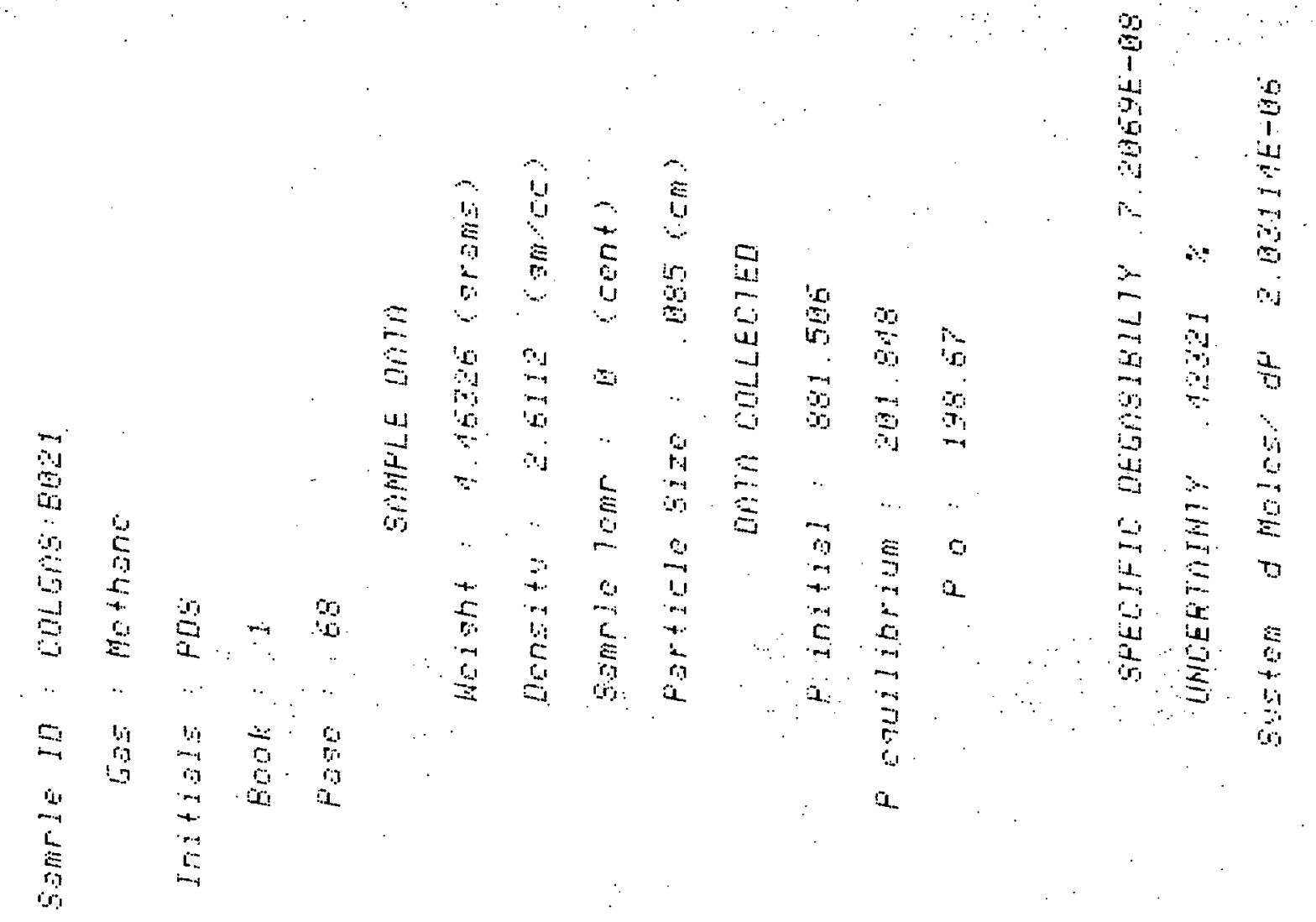




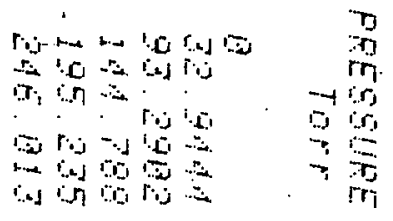

$\therefore$ and का

a 5 on

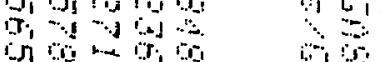

m r r r r r

$\$ 111$

का

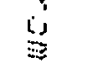

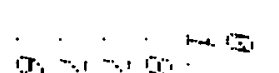

ar of 0

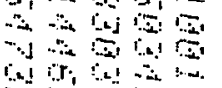

bit

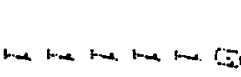

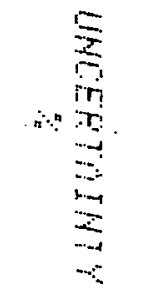

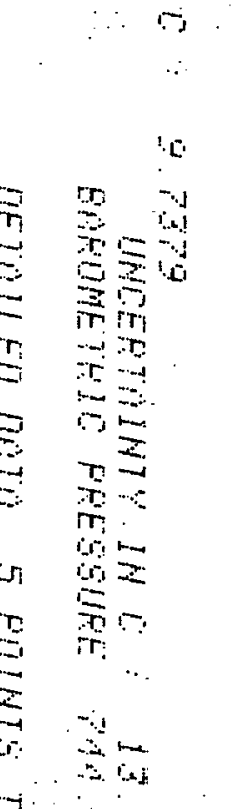

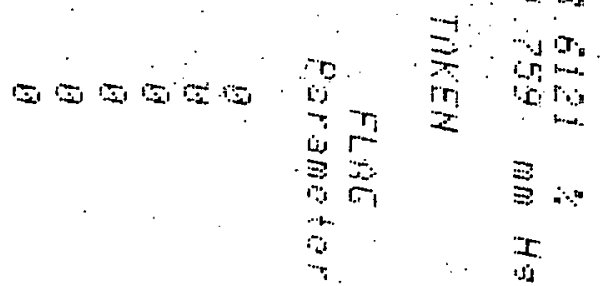

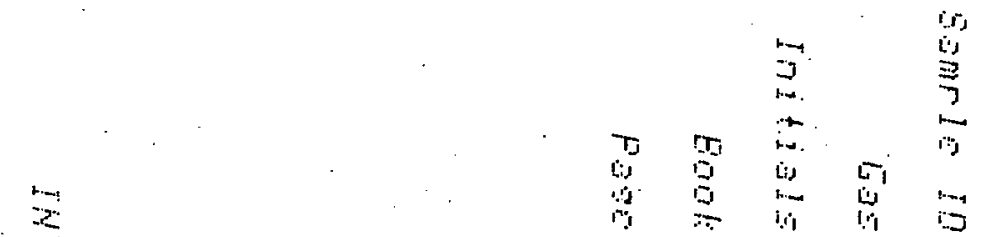

[.t] rit the ist

$9+2-1$

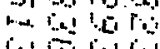

Tit

min

in

콜

1.

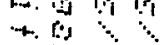

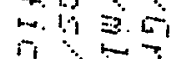

$\therefore \geqslant$

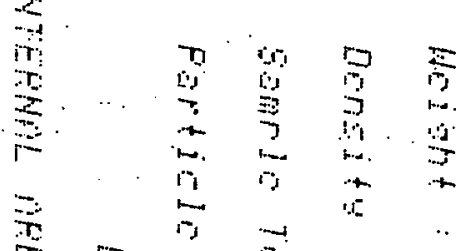

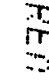

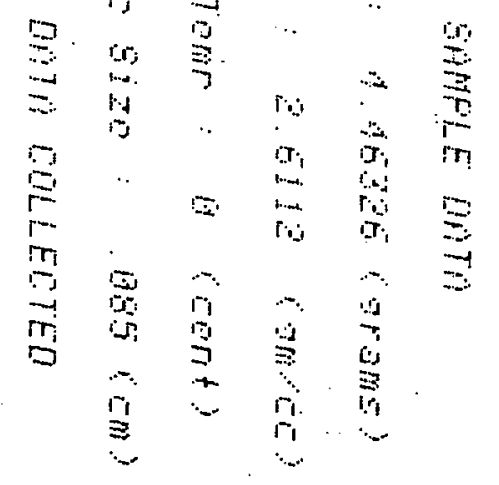


0
4
0
0
0
0
0
0
0
0
0

3
3
0
0
0
0

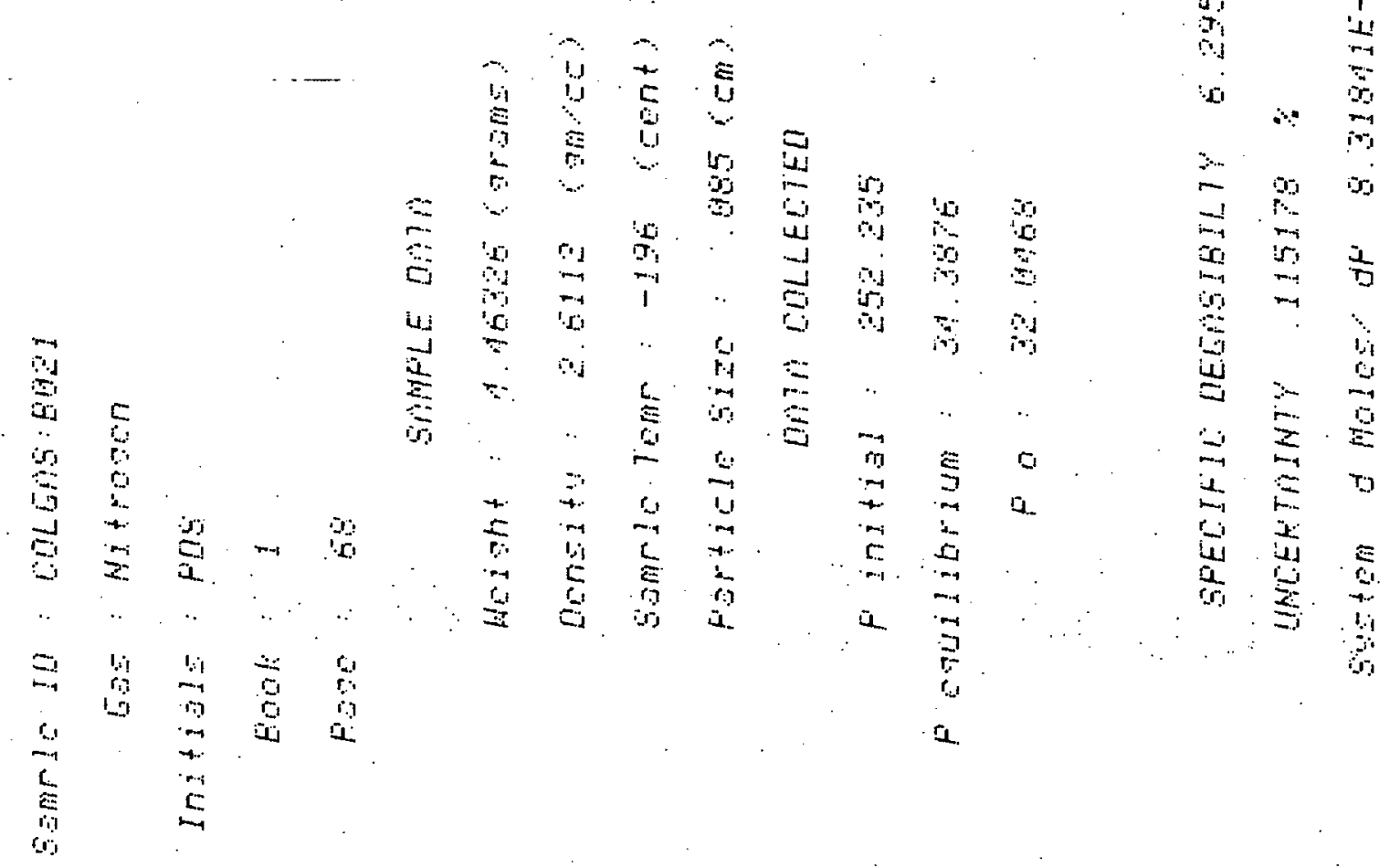




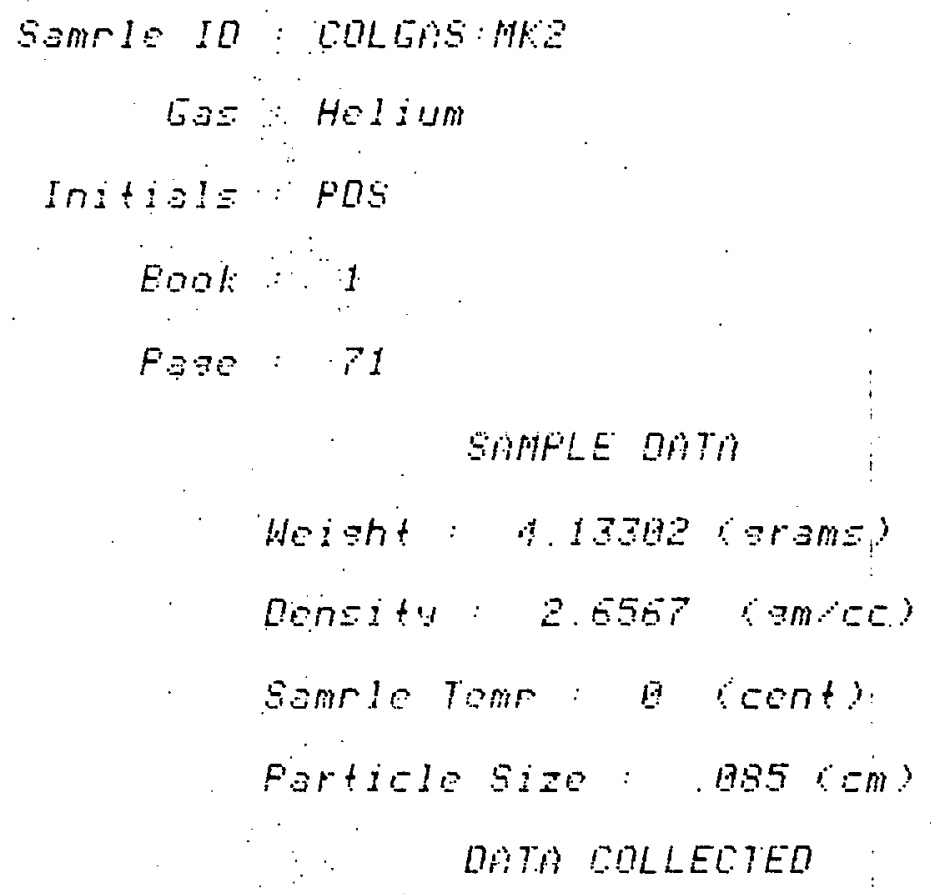

SLOFE UF ISOTHERM

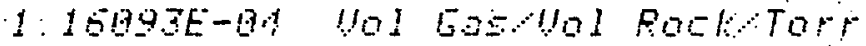

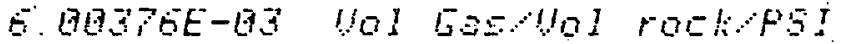

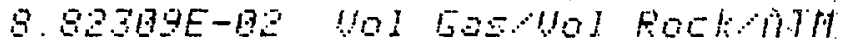

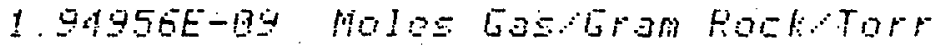

$$
\begin{aligned}
& \text { UNEERTAIHTY : Z. IBQTE }
\end{aligned}
$$$$
\text { DETAILEO DHTH " FUHHTS THEEH }
$$

FRESSUIRE

TOH

19.

236.563

522.861

815.651

885.391
ADS Gils

Molectorim

a $36 \mathrm{ABE}-\mathrm{B}$

․ $11820 E-B 7$

1. $6501 E-160$

1. $F B O A E-B 0^{\circ}$
UNEERTAIHT\%

$\because$

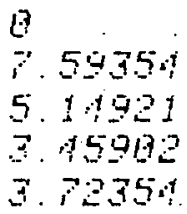

FLOE

LLAE

Pargneter 


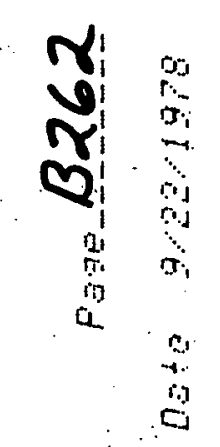

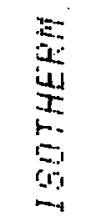

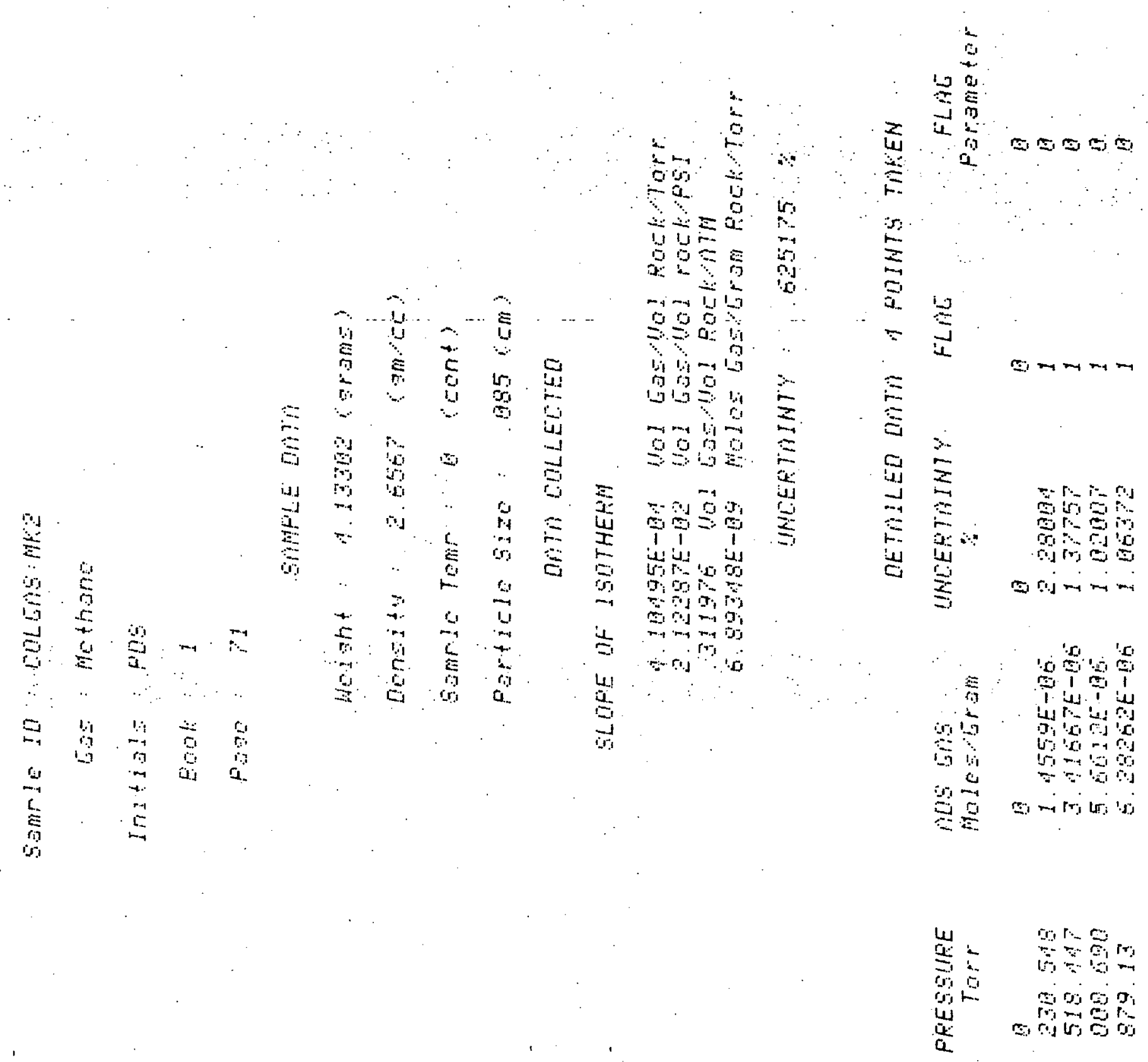




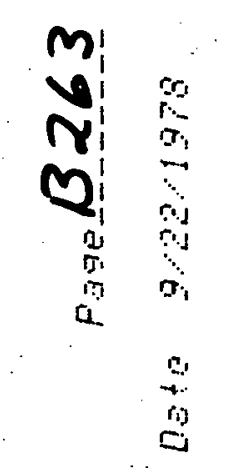

2
0
0
0
0
0
0
0

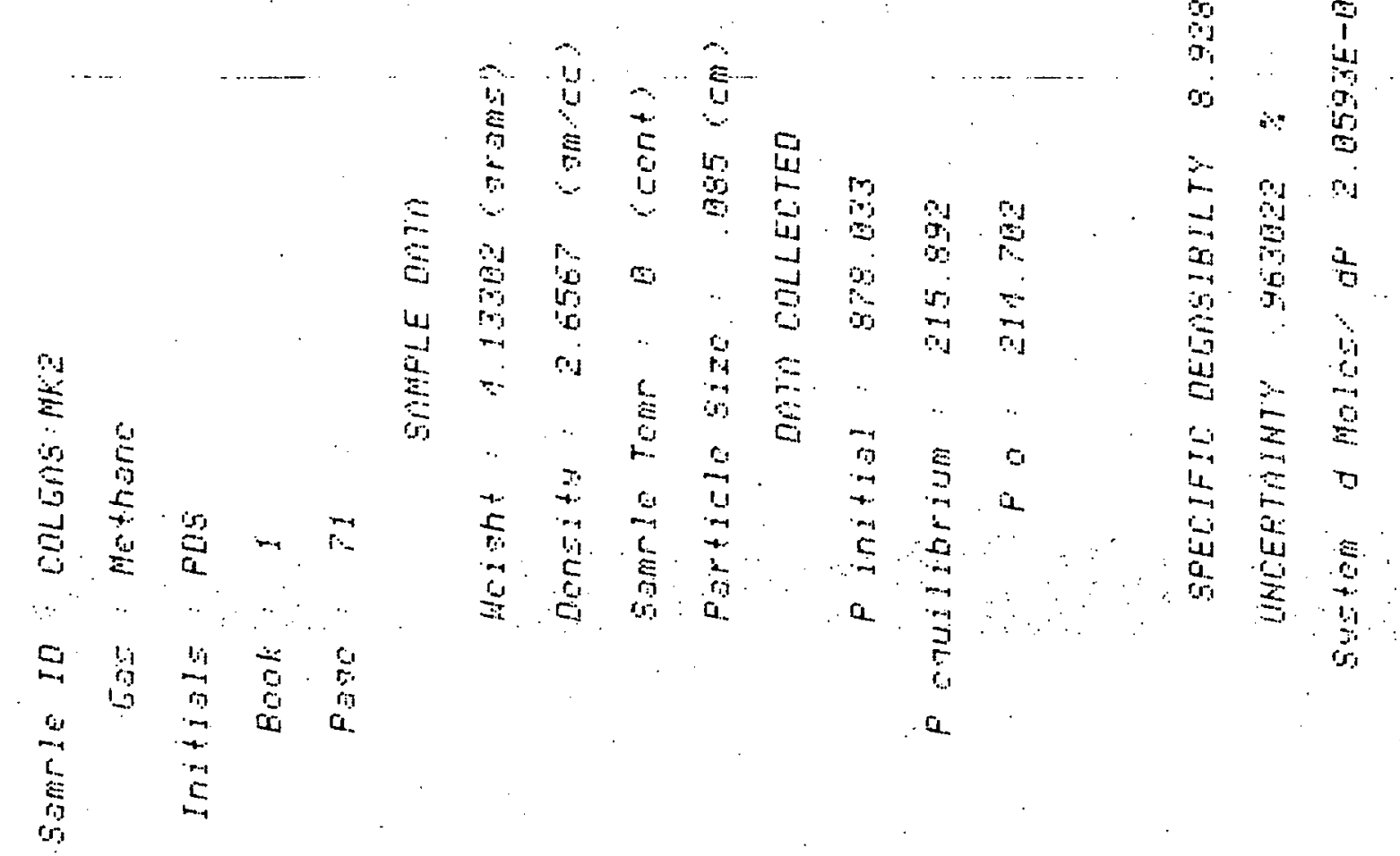




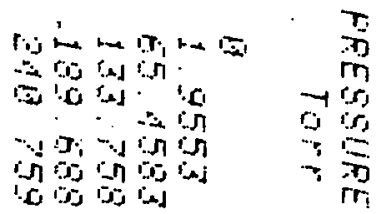

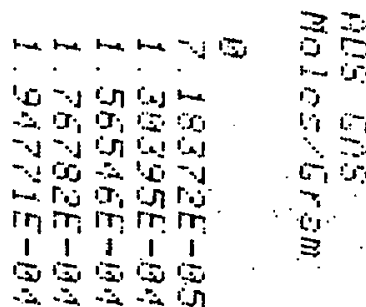

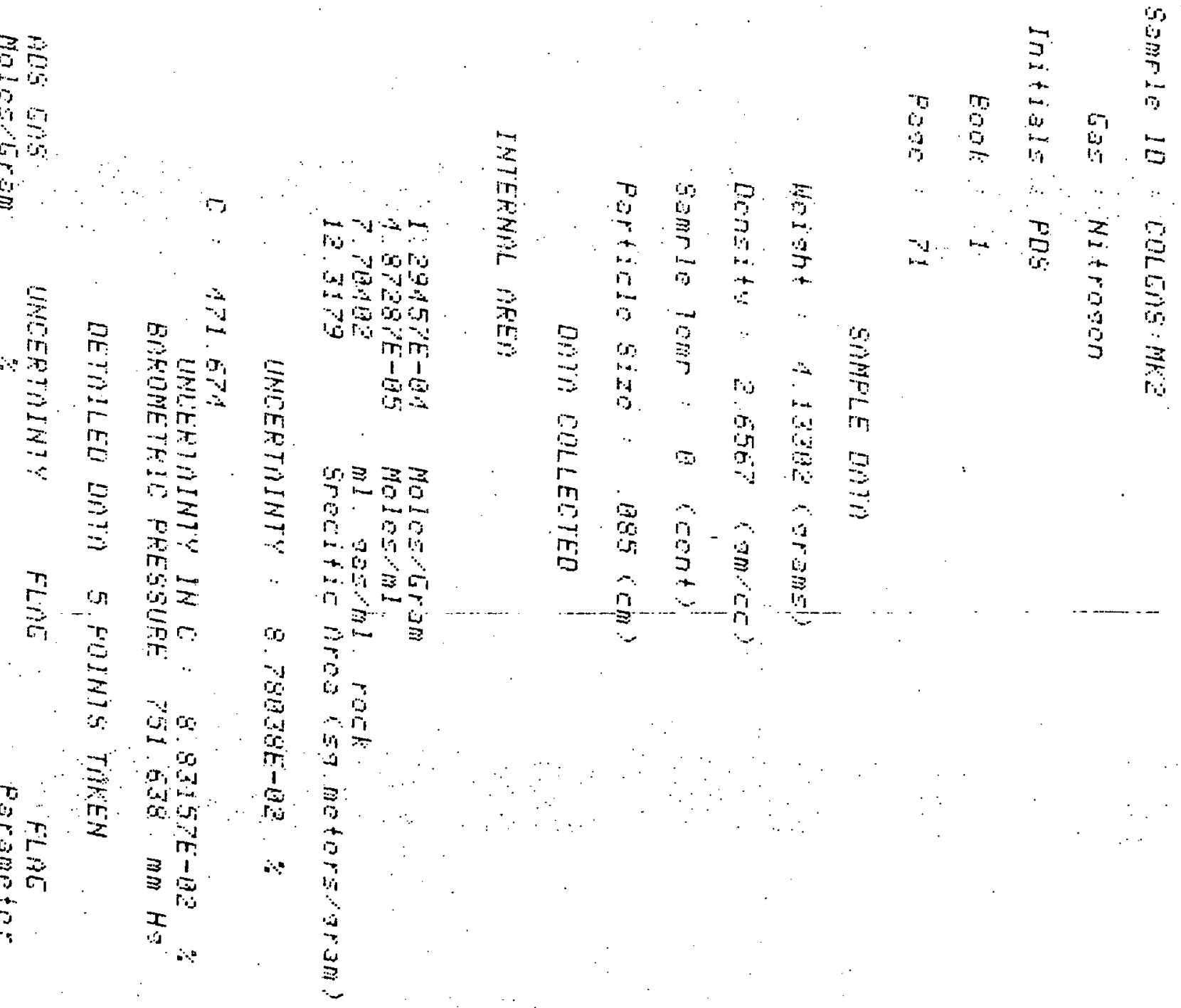




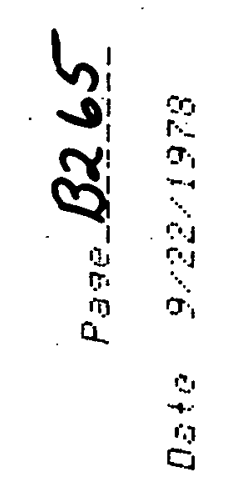

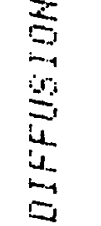

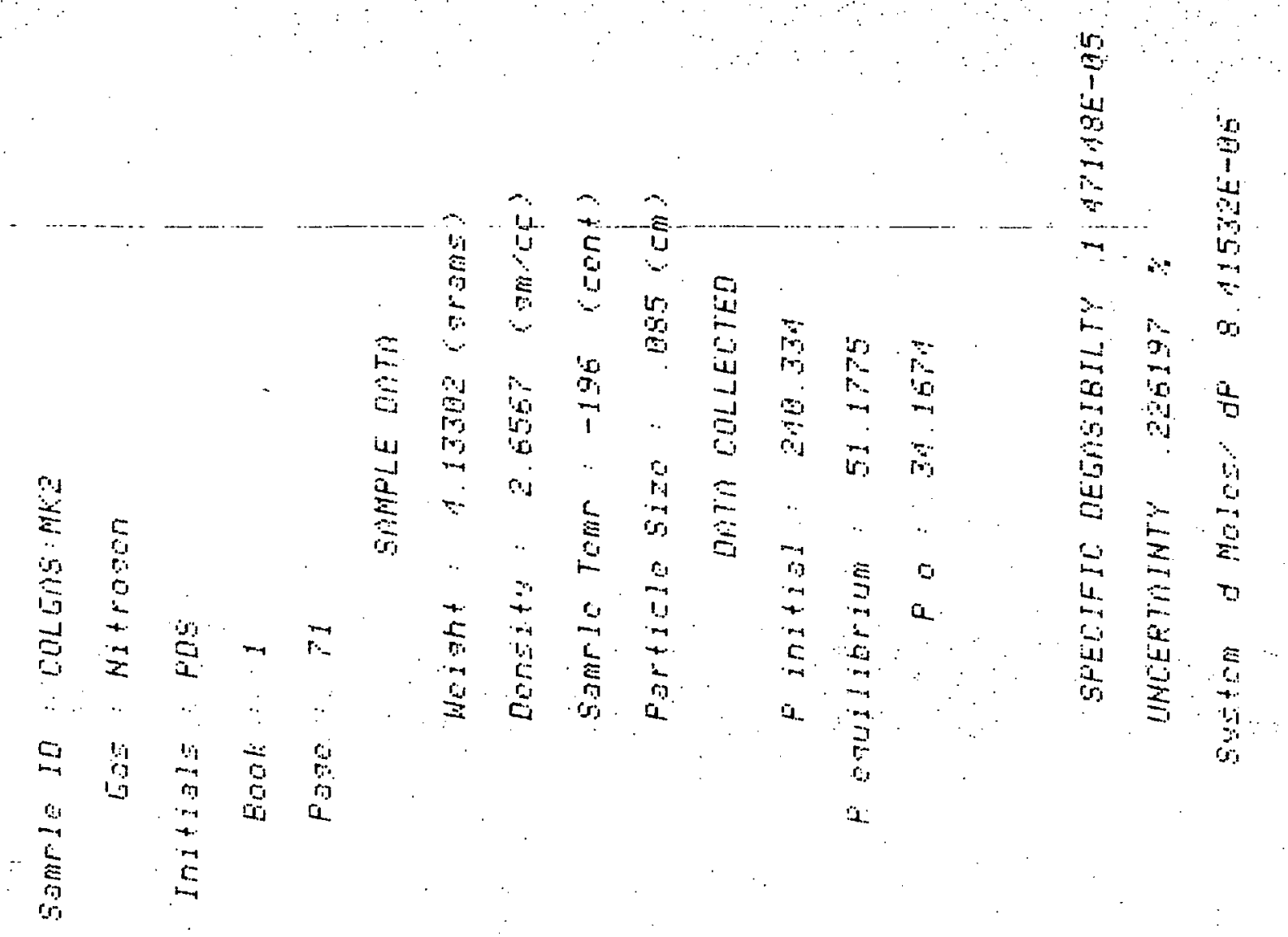




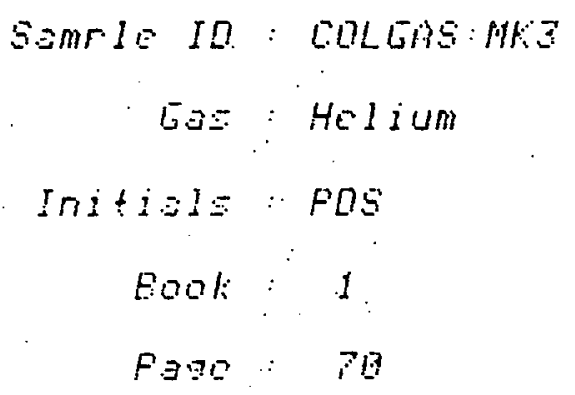

SHAPLE DATH

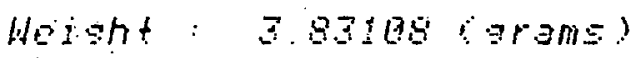

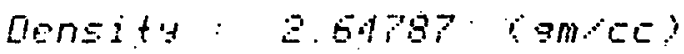

Semrlo Temr \& G cent?

Farticiesizo: . 055 (cm)

DATH COLLECTER

SLOFE OF ISOTHERM

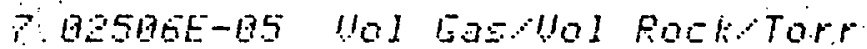

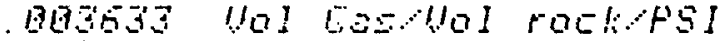

5. 33965E-BO Wo! GasUal RockATH

1.18305E-69 Moles Gastram FoskTort

UNEERTIHTY : $3.86187 \therefore$

\section{DETHILED DOTA \& POIHTS THKEH}

PRESSURE

Torr

19

2.99 .22

525.795

817.455

885.776
905695

Moles: $5 \mathrm{ram}$

19

2. PSBZSE-BO

5. $1895 E-67$

1. $191 \mathrm{TE}-1 \mathrm{BO}$

1. $19737 F-190$
UHEERTAIHTY

$\because$

17

12.901

9.7969

5. 98094

อ. 59768
FLAE

FLAE

Parsinetar

B

B

a

13

Q 


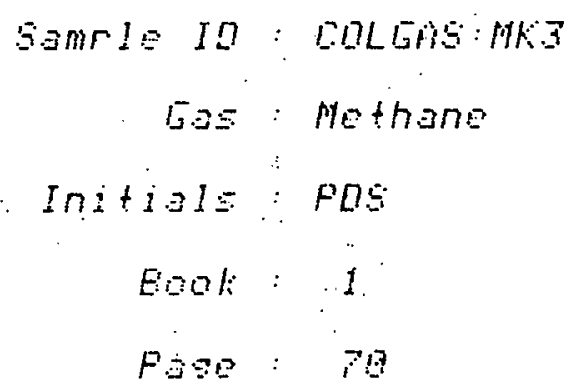

SAMPLE DHTH

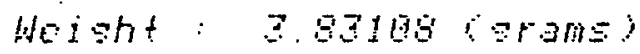

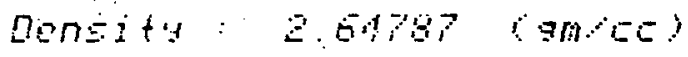

Sonrla Term : a ceont)

Paticlesico: . 085 (cm)

DOTTA COLLEETEO

SLOFE OF ISOTHERM

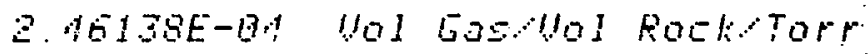

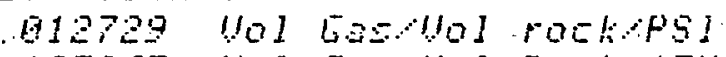

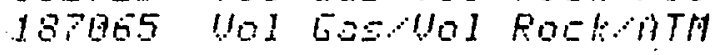

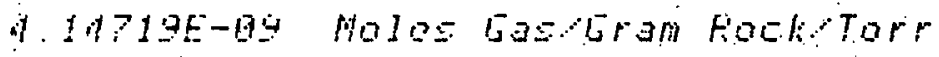

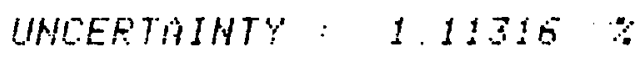

DETHILE DATH \& FOIHTS THKEH

FFESSIIFE

Torr

무

237.428

522.672

015.555

991.527
ADS Gns

holestorm

$\underline{9}$

B. $9999 \dot{E}-197$

2. BQSBJE-1A

3. $49.718 E-196$

3. $77693 E-80$
UNEEPTAJHIY

$\because$

보요

7. 98349

2. 52842

1. 78291

1. 90679
FLDE

FLA

$\theta$

1

1

1
FLAE

Pjometer

$\theta$
$\theta$
0 


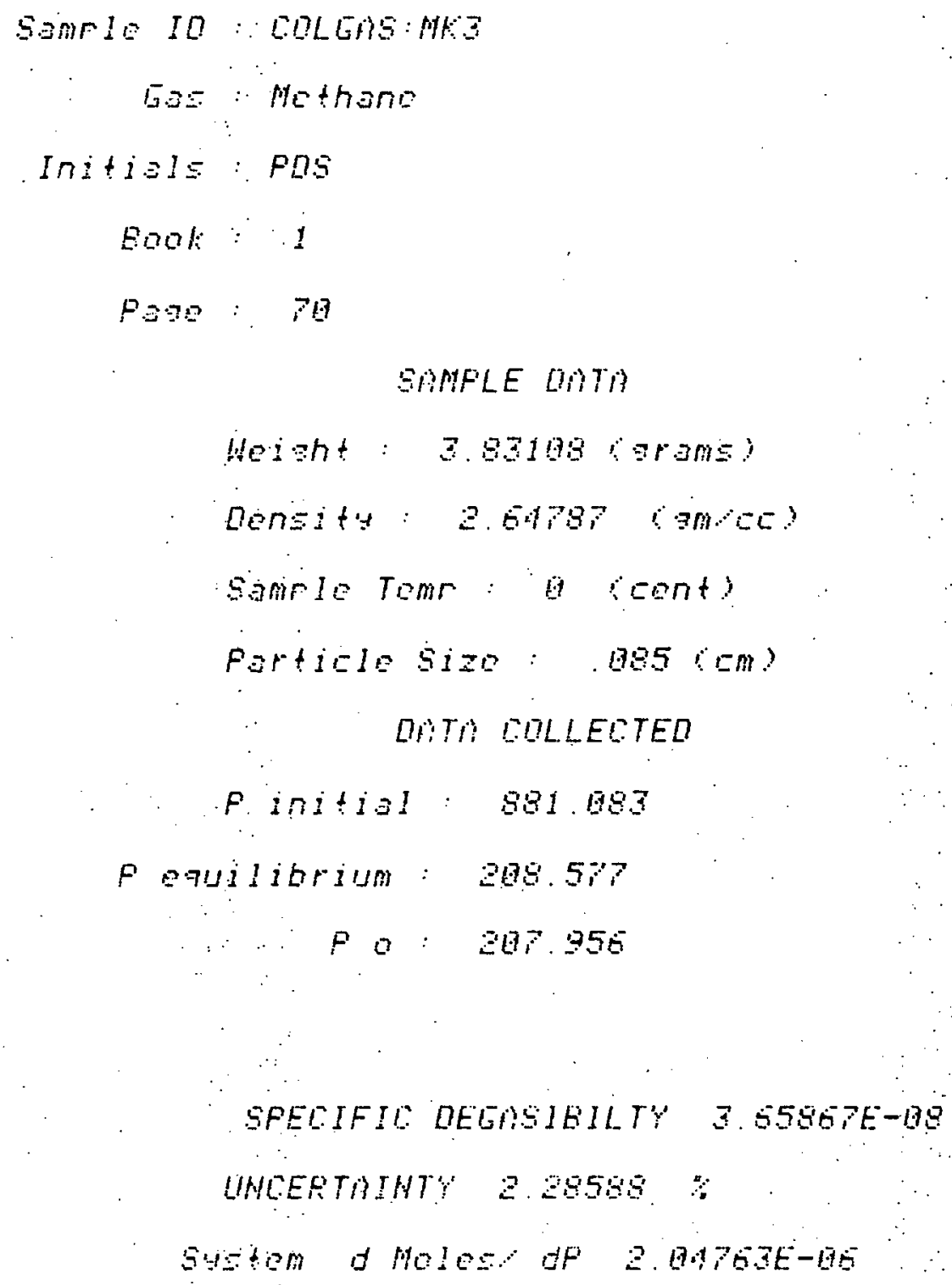




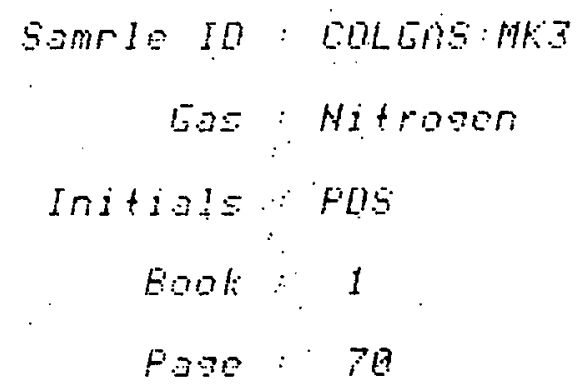

PRESSURE

Tor

Q

12.8912

84.796

142.735

$-192.195$

242.212

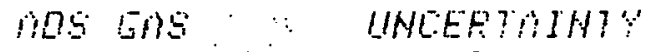

MoIn:

19 $\quad 0$

E. $20798 E-65 \quad .261604$

G. $7875 E-195$. 25102

1. $1136 E-B d \quad 250180$

1. $273 E-194 \quad .258802$

1. $37 P E-6 \cdot \quad 250222$
FLAE

FLAG

Parameto: 


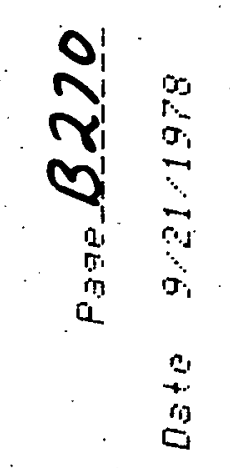

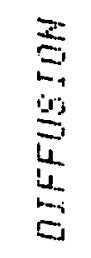

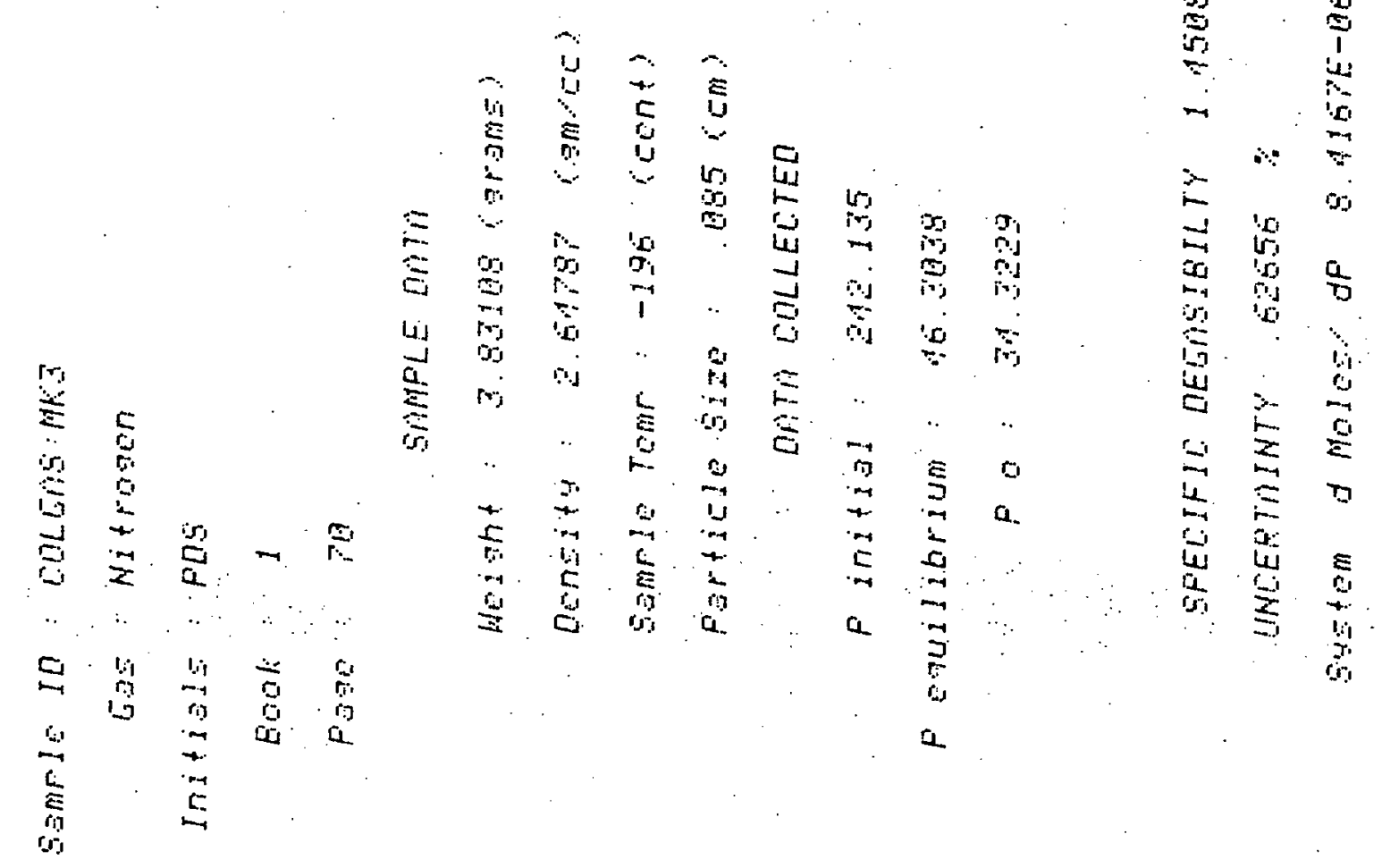




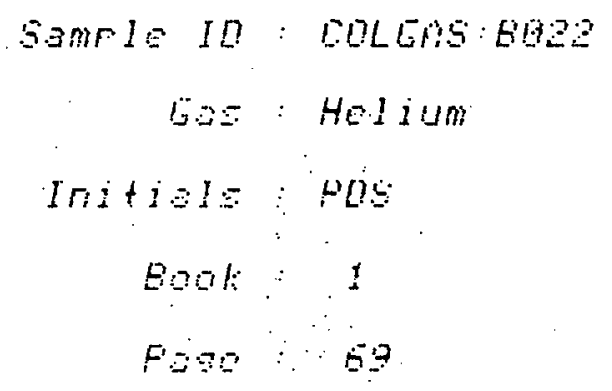

SAMPLE MU?

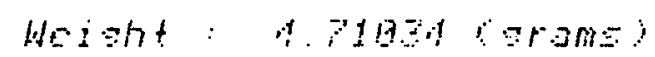

Donsty a zas amea?

Semrlo Tomr: la cent?

Porticlosizo: $895(\mathrm{~cm})$

DATA COLLETER

SLOPE OF IODTHENH

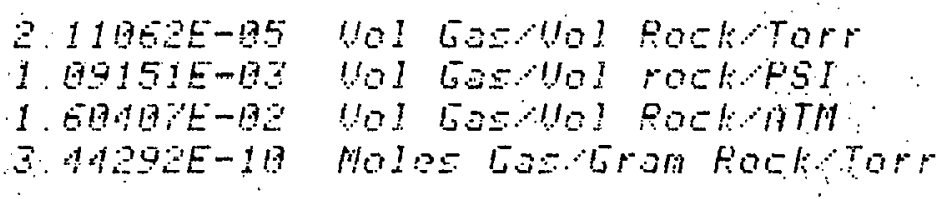

UINEERTAIHTY : $19.7 B 53 \%$

DETHLEO LITTH A POHTS THESH

PFESSUEE

b

230.553

520.117

017.752

$805.5 \%$
MIS: 50

Mot 0 -

19. $5221 E-198$

G. $4020 E-198$

3 $62709 E-19$

3. $959095-17$
UACELTAIH? $\because$

FLAO

9

198 . 190

18.1965

1.7. 94.4

1.7675
FLAG

Parameta

19

19.

B

19 


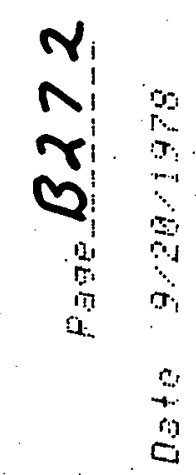

3
$\vdots$
0
5
5
$a$
$a$

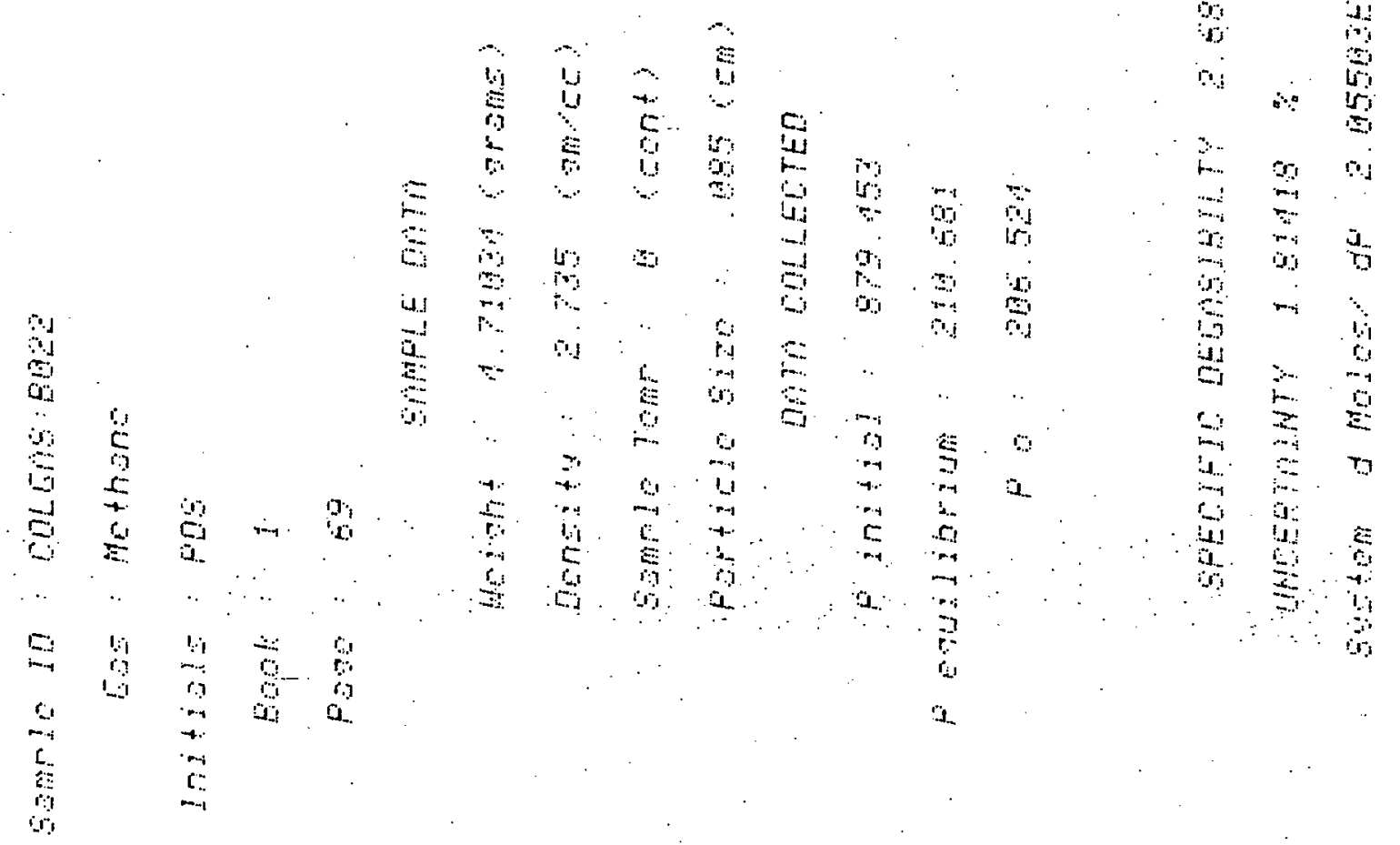




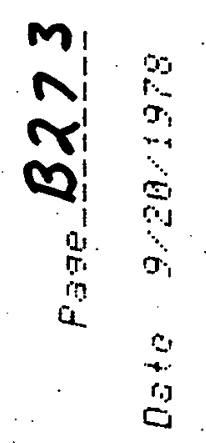

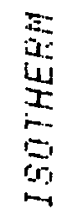

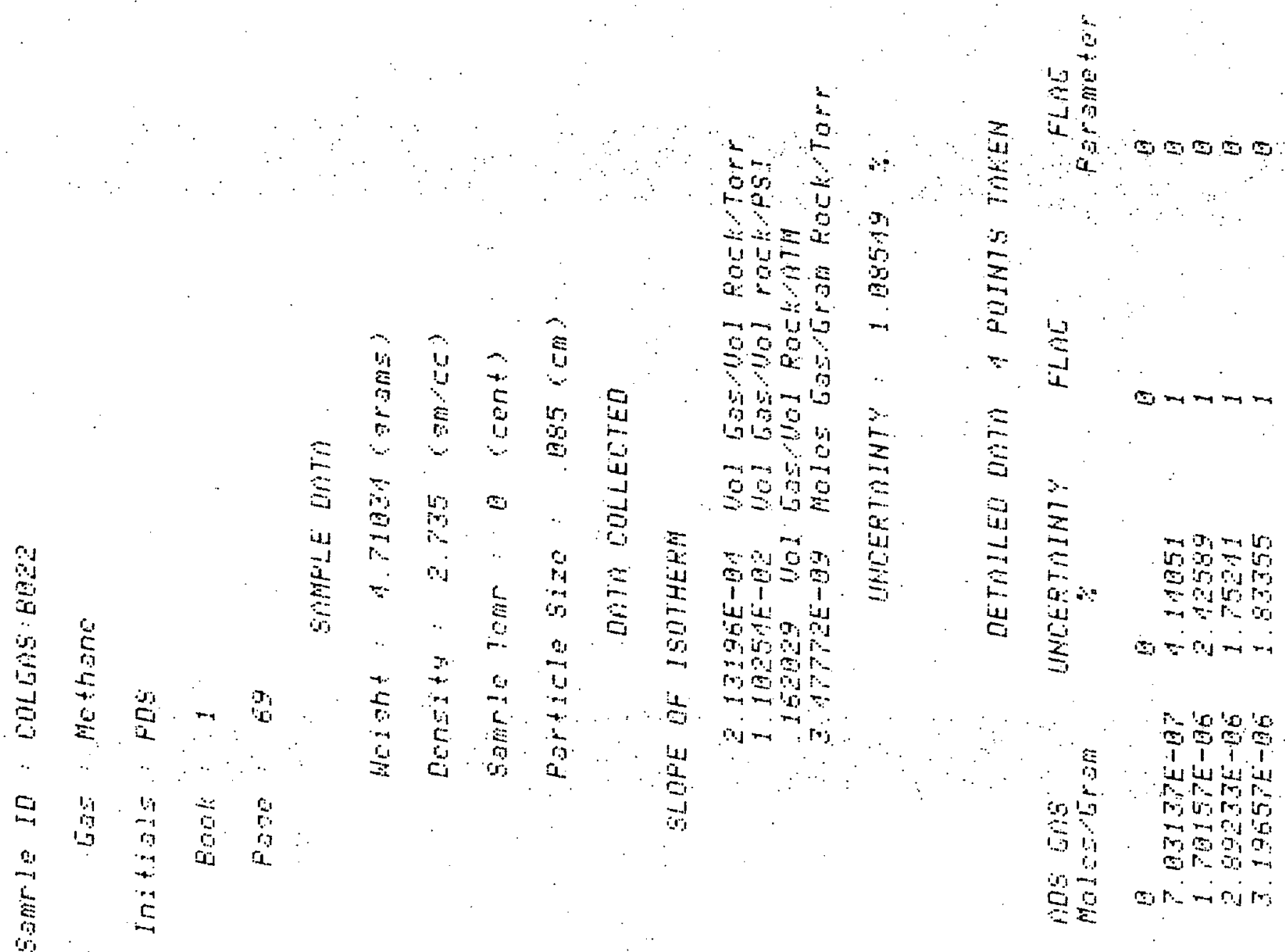

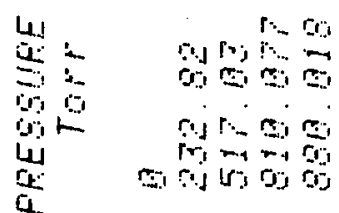



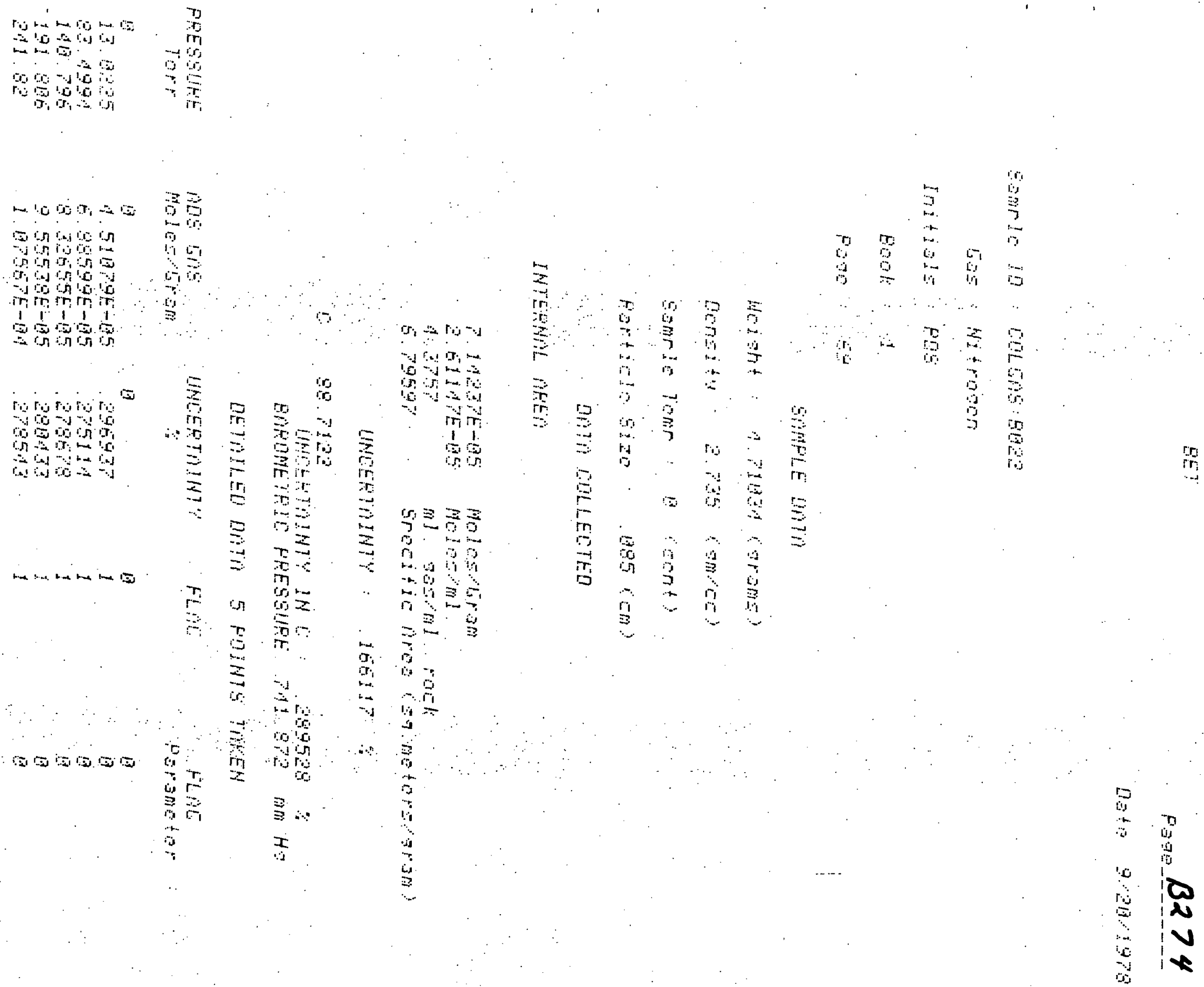


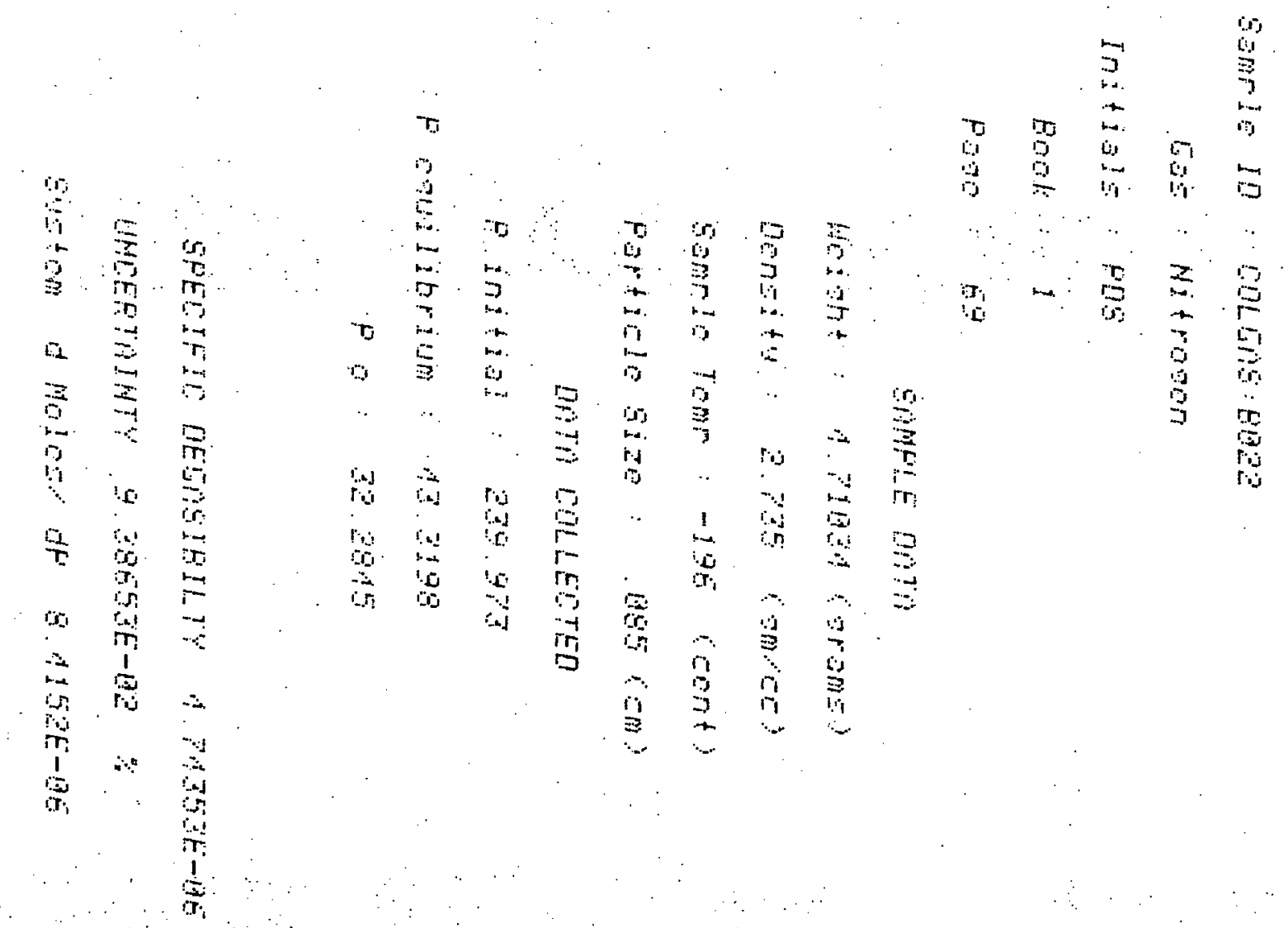


FILE\# $23-2: 3$

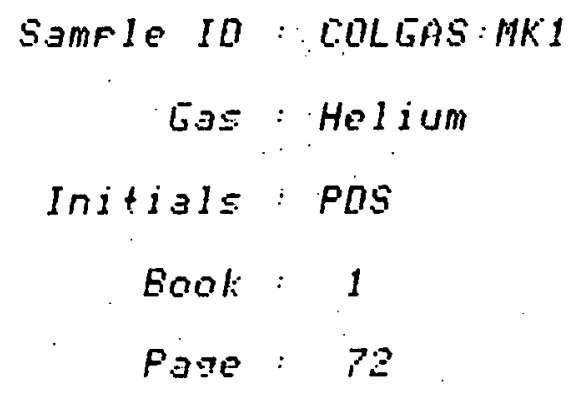

SAMPLE DATA

$$
\begin{aligned}
& \text { Weight : 4.31258 (grams) } \\
& \text { Density : } 2.6422 \text { (amlec) } \\
& \text { Sumrle Temp : a (cent) } \\
& \text { Particle size: } 0.085(\mathrm{~cm}) \\
& \text { DATA COLLEETEO }
\end{aligned}
$$$$
\text { SLOFE ITF ISOTHERM }
$$$$
\text { 7.51536E-195 Wol GasNUol Rock Torr }
$$$$
3.88656 E-193 \text { Wol Gas. Wol rock.PSI }
$$$$
\text { 5.71167E-192 Uol Gas Ullol Rock ATIA }
$$$$
\text { 1. 26899E-199 Moles Gas Gram Racklorr }
$$$$
\text { LINEERTAINTY : } 3.1733 \%
$$

DETAILED DATA 4 POINTS TAKEN

FRESSURE TORP

$$
B
$$

244.749

539.253

823.0995

889.993
ADS GAS

Molestorsm

UNCERTAINTY $\because$

FLAG
(3)
12. 5571
8. 136193
4. 79162
5. 28409
2. $5235 E-177$
$5.51495 E-197$
B
a
a
1. $14893 E-196$
1. $28486 E-86$
1
1
1
1

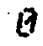
$\theta$
B

FLAE

Farameter 
FILE\# $23-2: 3$

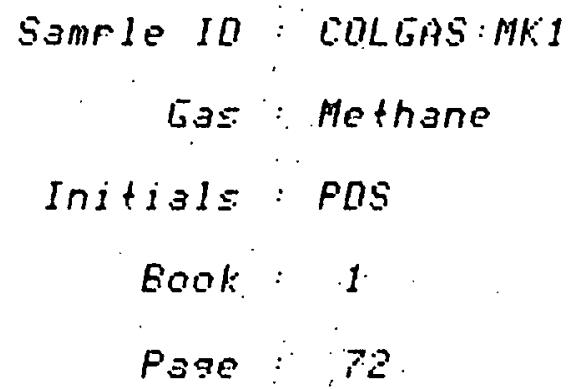

SAMPLE DATA

Weight : 4.31258 ipramsy

Density : 2.6422 (amere?

Sample Temr: to (cent)

Particle sizo: $085(\mathrm{~cm})$

DATA COLLECTER

SLOPE OF ISOTHERM

2:554B2E-04 Wol Gas Nol Rock Tor

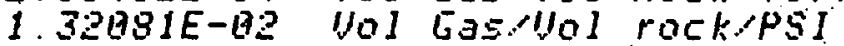

1941 as. Wol Gas Wol RockntA

4.31252E-199 Moles Gas Lram Rockitorr

UNEERTAINTY : .924238:

DETAILEO DATA A PUIHTS THKEN

\section{FRESSLIRE}

Torr

6

260.832

541.021

821.154

888.756
ADS GAS

Molesuram

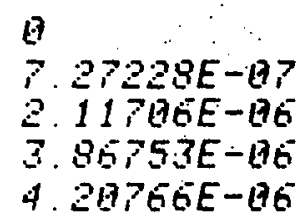

UNCERTAINTY $z$

FLAE

$\theta$

4.36487

2. 12557

1. 42852

1. 51828
FLAE

Perameter
a
a
Q
B
a 


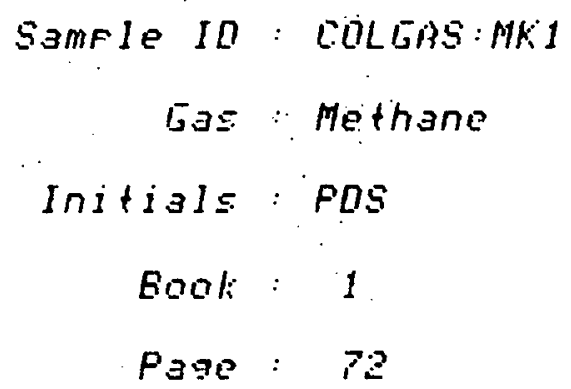

SAMPLE DATA

Height : 4.31258 (arams?

Density : 2.6922 (am 00 )

Samrle Temr : a (cent)

Particle size: $095(\mathrm{~cm}$ ?

DATA COLLECTED

Finitial $\therefore 888.012$

$P$ equilibrium : 204.29

$F_{0}: 280.351$

\begin{abstract}
SPECIFIO DEGASIBILTY 1.21983E-Q7?
UNCERTAINTY : 677794 $\because$
\end{abstract}

System a Molos dP 2. B2P2AE-166 


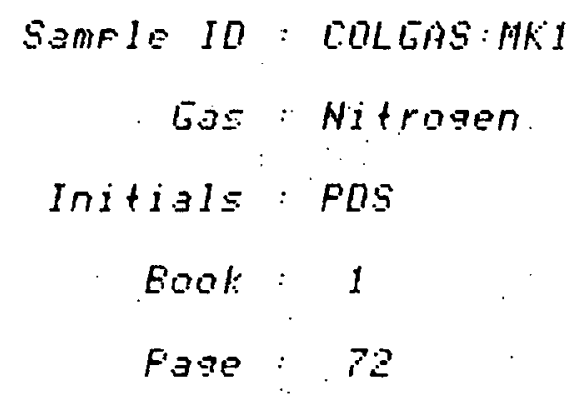

SAMPLE DATH

Weight : 4. 31258 (arams?

Density : 2.0.22 (9mac)

sample Temp: a (cent)

Farticle size: $.085(\mathrm{~cm})$

DATA COLLECTEO

INTERNALL AREA

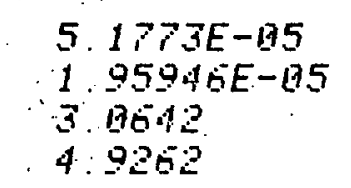

Molestram

Molesoml.

ml. $9 a \leq \mathrm{ml}$. rock

Srecjfic hres ceqmetersaran?

$$
\text { UNCERTAINTY : . } 192933 \%
$$

C. 22.9997

UNCERTAINTY IN $C: 2.44 P B A$

BARUAETRIL PRESSURE TA5.7BO $\mathrm{mm} \mathrm{HO}$

DETAILED DATA 5 POINTS TAKEN

FRESSLIRE

Torer

봉

25.9981

89.9705

142.938

192.818

244.962
ADS GAS

Molestram

日

2. $52588 E-05$

4. $18919 E-05$

5. $24791 E-05$

o. $315.15 E-15$

7. G7PE-QS5
UNCEFTAINTY

$\because$

8

.579388

503559

182955

463143

443584
FLAE

日

1

1

1

1
FLAG

Parameter 


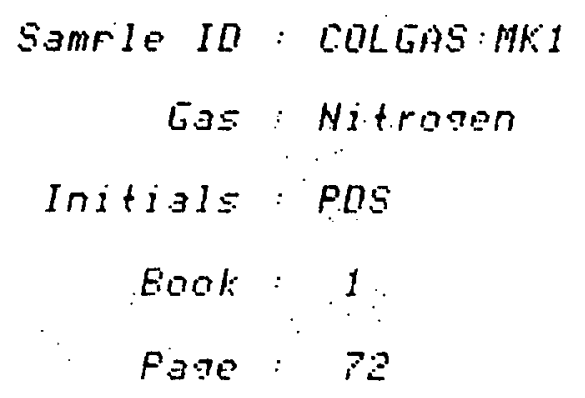




\author{
Sample ID: COLGAS:MKA \\ Gз5 : Helium \\ Initials: POS \\ Book: : 1 \\ Page : 73
}

SAMPLE IATA

$$
\begin{aligned}
& \text { Weight : 1.96204 forams? } \\
& \text { Density : 2.6.17 (amer) } \\
& \text { Somple Temp : } 0 \text { (cent) } \\
& \text { Particle sizo: } 085(\mathrm{~cm}) \\
& \text { DATA COLLECTED }
\end{aligned}
$$

SLOFE OF ISOTHERM

\begin{tabular}{|c|c|c|c|c|}
\hline $\begin{array}{c}\text { PRESSURE } \\
\text { Tarr }\end{array}$ & $\begin{array}{l}\text { ADS GAS } \\
\text { MOLPSEr Gm }\end{array}$ & $\begin{array}{c}\text { UNEEFTAINTY } \\
\because\end{array}$ & $F L A G$ & $\begin{array}{l}\text { FLAE } \\
\text { PErame ter }\end{array}$ \\
\hline $\begin{array}{l}9 \\
237.658 \\
525 \cdot 216 \\
815.575 \\
884.285\end{array}$ & 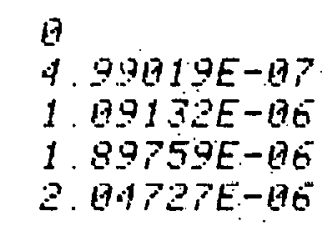 & $\begin{array}{l}\text { 9. } 528196 \\
\text { 3. } 58687 \\
\text { 2. } 52640 \\
\text { 2. } 79655\end{array}$ & $\begin{array}{l}0 \\
1 \\
1 \\
1 \\
1\end{array}$ & $\begin{array}{l}19 \\
0 \\
0\end{array}$ \\
\hline
\end{tabular}

$$
\begin{aligned}
& \text { 1. 32971E-194 Uol GasNol Rock Torr }
\end{aligned}
$$

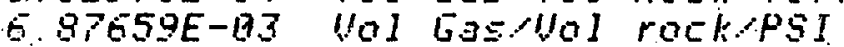

$$
\begin{aligned}
& 191958 \text { Vol GasNol RocksatA } \\
& \text { 2. 24567E-Q79 Holes Gasuram Rock Torr } \\
& \text { UNCERTAIHTY: } 1.575 \% \%
\end{aligned}
$$

DETAILEE RATA A POIHTS TAKEN 
FILE\# $23-2: 4$

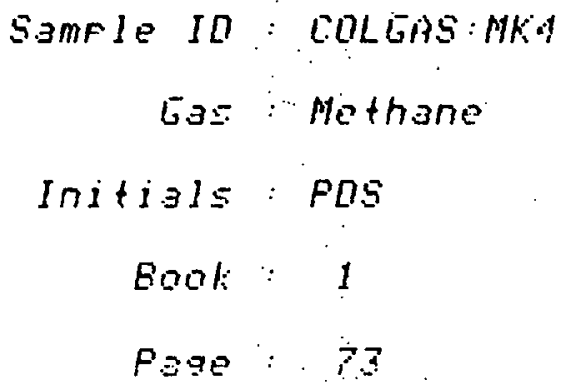

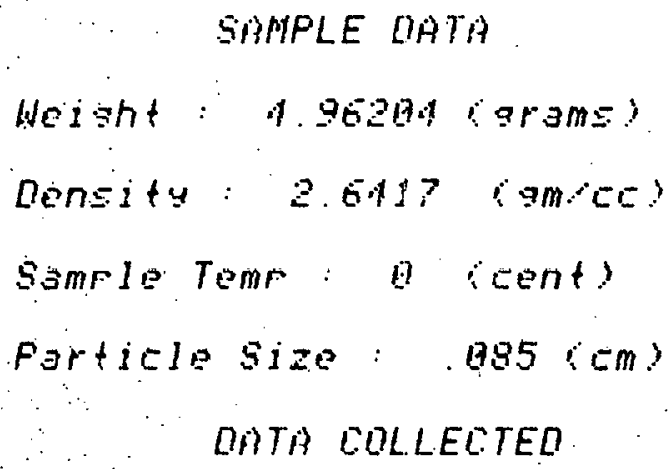

SLQPE UF ISOTHERH

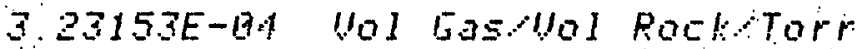
1. GT119E-6Z Ual Gas Vol rockPSI 245596 Vol GasulNol Rocklth

5. 45755E-B9 Molos Gastiram RoEk Torr UNCERTAINTY : . ES5STI $\%$

DETAILED DATA 4 POINTS TAKEN

\begin{tabular}{|c|c|c|c|c|}
\hline $\begin{array}{c}\text { PRESSUIRE } \\
\text { TOFP }\end{array}$ & $\begin{array}{l}\text { ADS laAs } \\
\text { Moles laram }\end{array}$ & UNCERTAINIY & $F L A G$ & $\begin{array}{l}\therefore F L A G \\
\text { Porameter }\end{array}$ \\
\hline B? & 18 & 8 & $B$ & 8 \\
\hline 236.88 & 1. $\operatorname{lad} d 2 E-196$ & 2.524 .15 & 1 & 8 \\
\hline 510.34 & 2. MES5E-BE & 1. 4203 & 1 & $\theta$ \\
\hline 817.535 & A. $529 B A E-190$ & 1. 86207 & 1 & B. \\
\hline 891.75 & 4. 961935E-170 & 1. 12136 & 1 & $\theta$ \\
\hline
\end{tabular}




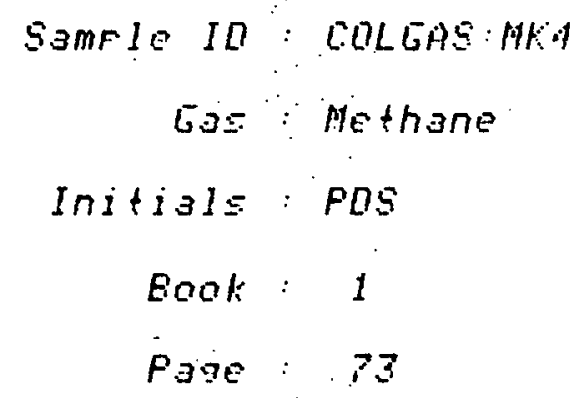




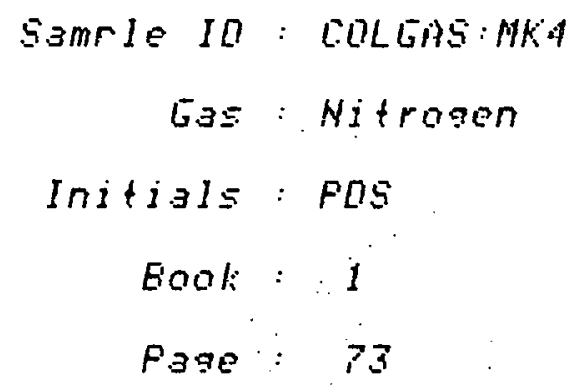

SAMPLE DATA
Woight : 1.96204 forams?
Denity : 2.6417 (ambe)
Ssmrle Temr: a icent)
Particlesize: $085(\mathrm{~cm}$ ?
DATA COLLEGTED

INTERNAL AREA
$9.83521 E-175$
3. $73306 E-195$
5. 8199
9. 35821

Molesogram

Moles.m.l

ml. gasoml rock.

Srecitic hros asqmetersoram?

$$
\text { UNCERTAINTY : 9.969PIE-B2 } 8
$$

i. : 238.954

UNCERTAINTY IN C: 1 : $2903 \%$

BAROMETFIC PRESSURE TAT QOQ $\mathrm{mm} \mathrm{HO}$

DETPILEU DATA 5 POINTS TAKEN

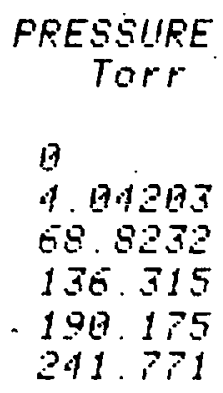

ADS GAS

Molesteram

UNCERTAINTY

$\because$

FLAE

FLAG

19

$5.01333 E-195$

9. $952805-195$

1. $18721 \mathrm{E}-174$

1. $32965 E-0.4$

1. $40607 E-64$

a.

220517

18009

185544

191314

193931

0
1
1
1
1
1

0
0
0
0
0
0




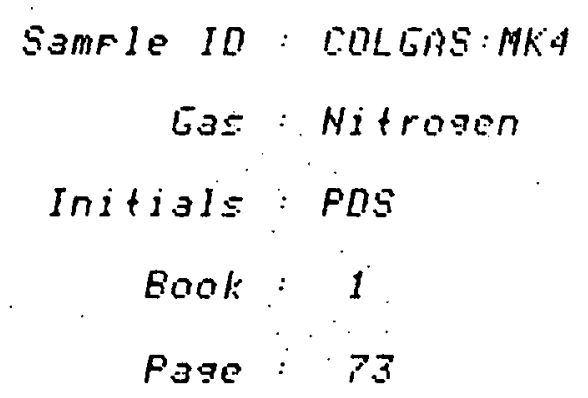

SAMPLE DATA

Height : 4.96204 (spams?

Density : 2.6417 (ameE)

Sample Temr: $-196^{\circ}$ (cent?

Particle sizo: 085 (Im)

DATA collegted

F initial: 242.389

$P$ equilibrium: $\quad 47.0423$

$F o: 33.3021$

SPECIFIC DEGASIBILTY 1.4211E-65

UINCERTAINT'Y .698523\%

system a Moles dP B.38979E-136 


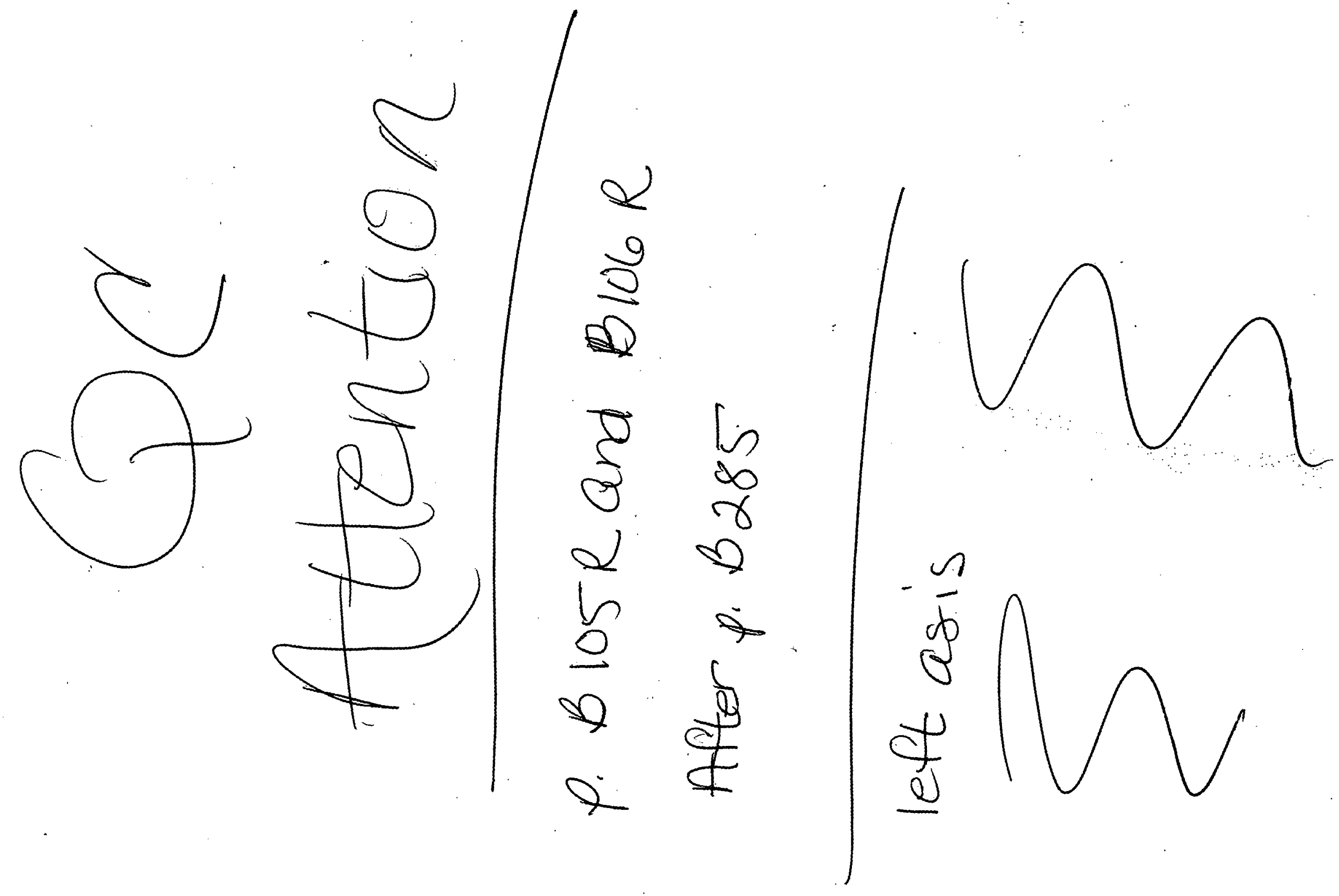


FILEH $22-1: 17$

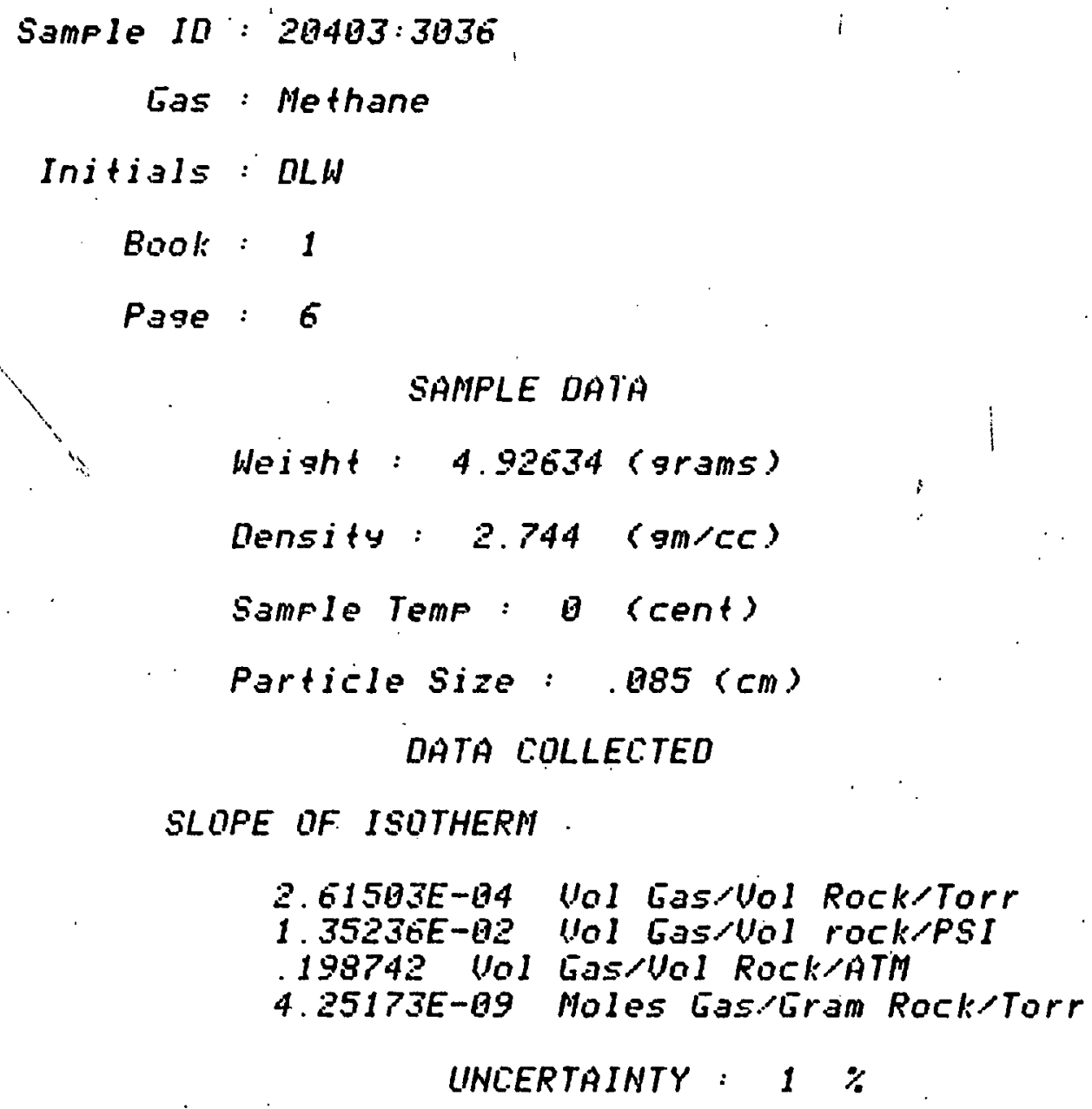

FRESSURE Torr 237.983 524.799 817.579 884.5
ADS GAS Molesitram

1. $0764 E-60$

2. $24416 E-106$

3. $44363 E-196$

3. $71525 E-190^{\circ}$
UINCERTAINTY $\%$

FLAG
2. 57747
1. 7518
1. 48995
150106

FLAG

Parameter
$\theta$
$a$
0
$B$ 
FILE\# $22-1: 17$

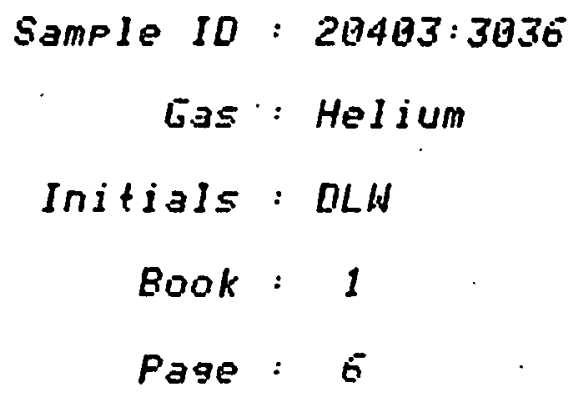

SAMPLE DATA

Weight : 4.92634 (grams)

Density : 2.744 ( $\mathrm{gm} / \mathrm{Cc}$ )

Sample Temp: $\theta$ (cent)

Particle Size: $.085(\mathrm{~cm})$

DATA COLLECTED

SLOPE IF ISUTHERM

$6.68738 E-195$

3. $45837 E-93$

5. $08241 E-172$

Wal Gas Wol Rock/Torr

1: $08729 E-09$

Ual Gas vol rockipsI

Ual Gasrvol Rock/ATM

UINCERTAINTY : $1 \%$

DETAILEO DATA 3 POINTS TAKEN

PRESSURE

Torr

242.343

539.856

824.241

$893 \cdot 133$
ADS GAS

Molestoram

2.81637E-Q7

5. 53159E-97

9. Q2192E-BP

9. $72565 E-07$
LINCERTAINTY

$\%$

9.83515

7.08938

5. 32938

5.71204
FLAG

Parameter 
ORO-5197-10

Appendix C

\section{SLAB DATA}

\section{Juniata College}

Huntingdon, Pennsylvania 16652 


\section{Legend and Explanations}

Date

Date sample was placed on apparatus.

Sample ID

We11 ID: depth in feet; suffix L - slab cut so that exposed faces are paralle 1 to bedding.

P - slab cut so that exposed surfaces

F - fracture. are perpendicular to bedding:

\section{Thickness}

Slab thickness.

Area

Total area of unmasked surfaces.

Pinitial

Pressure (torr) before pressure jump. Equilibrated for at least a week.

Pend of jump

Pressure (torr) at end of applied discontinuous pressure change.

Specific Degasibility

M1 gas degassed $/(\mathrm{sec})^{\frac{1}{2}} /$ torr $/ \mathrm{cm}^{2}$ area.

Uncertainty

Least square uncertainty of above measurement.

System d Moles/dP

Calculated system response in terms of 非 moles gas necessary to raise system.pressure 1 torr. 


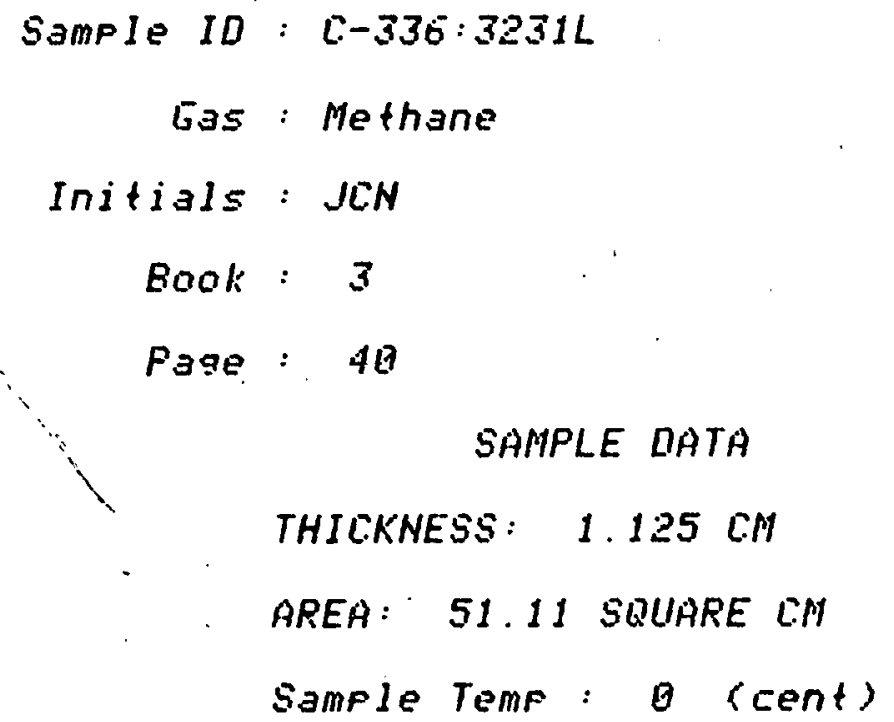

\author{
DATA COLLECTED \\ Finitial: 749.478 \\ $P$ end of iump: 340.132
}

SPECIFIC DEGASIBILTY $9.43273 E-86$

UNCERTAINTY $9.60649 E-02: \%$

sustem d Molest dP 1.71009E-05 


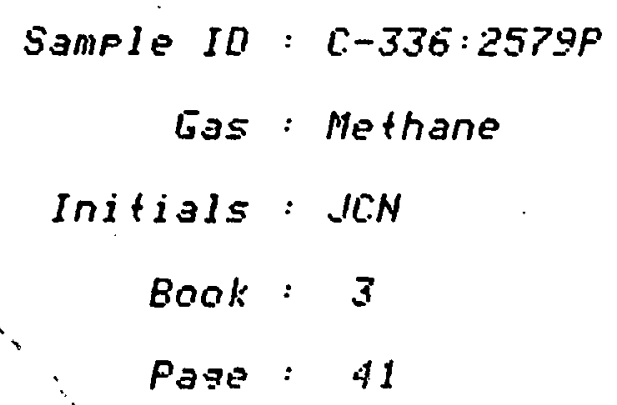

SAMPLE DATA

THICKNESS: O CM

AREA: 79.51 SQUARE LM

Sample Temp : $\theta$ (cent)

DATA COLLECTED

Pinitial: 745.874

$P$ end of iump: $176.610^{\circ}$

SPELIFIL DEGASIBILTY $9.23334 E-98$

UNEERTAINTY .1443:P $\approx$

System d Males, dP 9.41262E-176 


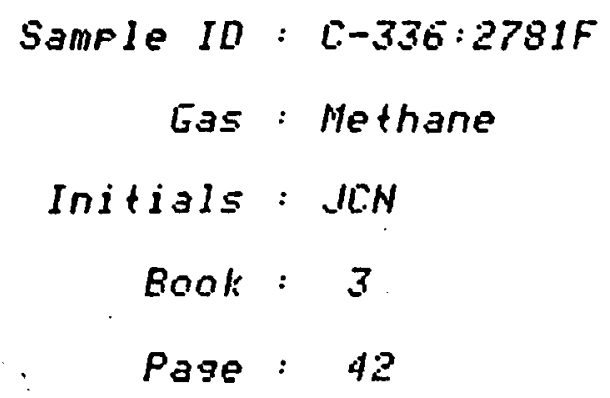

SAMPLE DATA

THICKNESS: $1.53 \mathrm{CH}$

AREA : 21.04 SQLIARE CH

Sample Temp : $a$ (cent)

DATA COLLECTED

$P$ initial: 746.268

$P$ end of jumf : 178.637

SPECIFIC DEGASIBILTY 3.22223E-B8

UNEERTAINTY . $13138 \% *$

System d Males dP $9.661990-60$ 


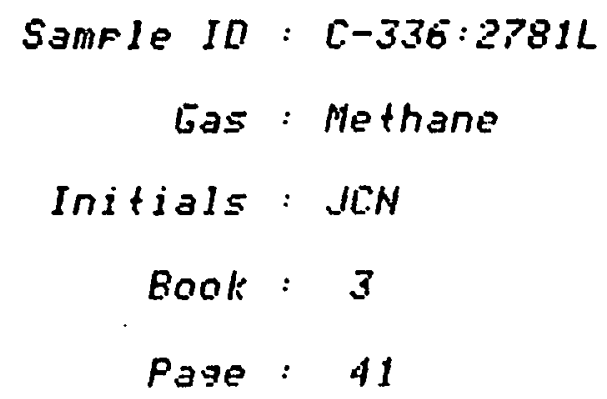

System d Males/ of 1, B8529E-095 


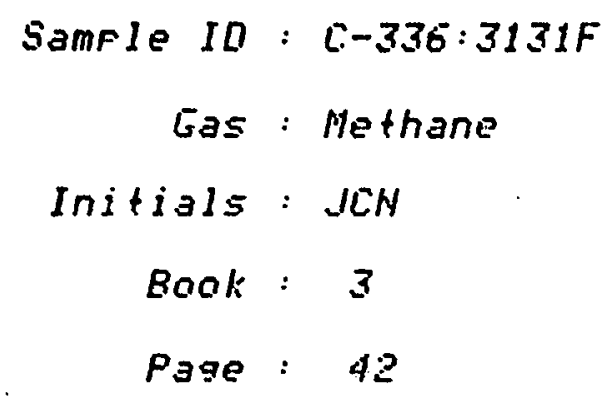

SAMPLE DATA

THICKNESS: 2 CM

AREA : 51.24 SRUAARE LA

Sample Temr: 1 (cent)

DATA COLLECTEO

$F$ initial: 745.303

$P$ end of iump : 220.759

SPECIFIL DEGASIBILTY $1.21399 E-B P$

UINEERTAINTY $1.49094 \%$

Sustem o Moles aP 1.07P95E-05 
OR0-5197-10

Appendix D

THE ESTIMATION OF RESERVES IN TIGHT SHALES

Paul D. Schettler, Jr.

Juniata College

Huntingdon, Pennsylvania 16652 


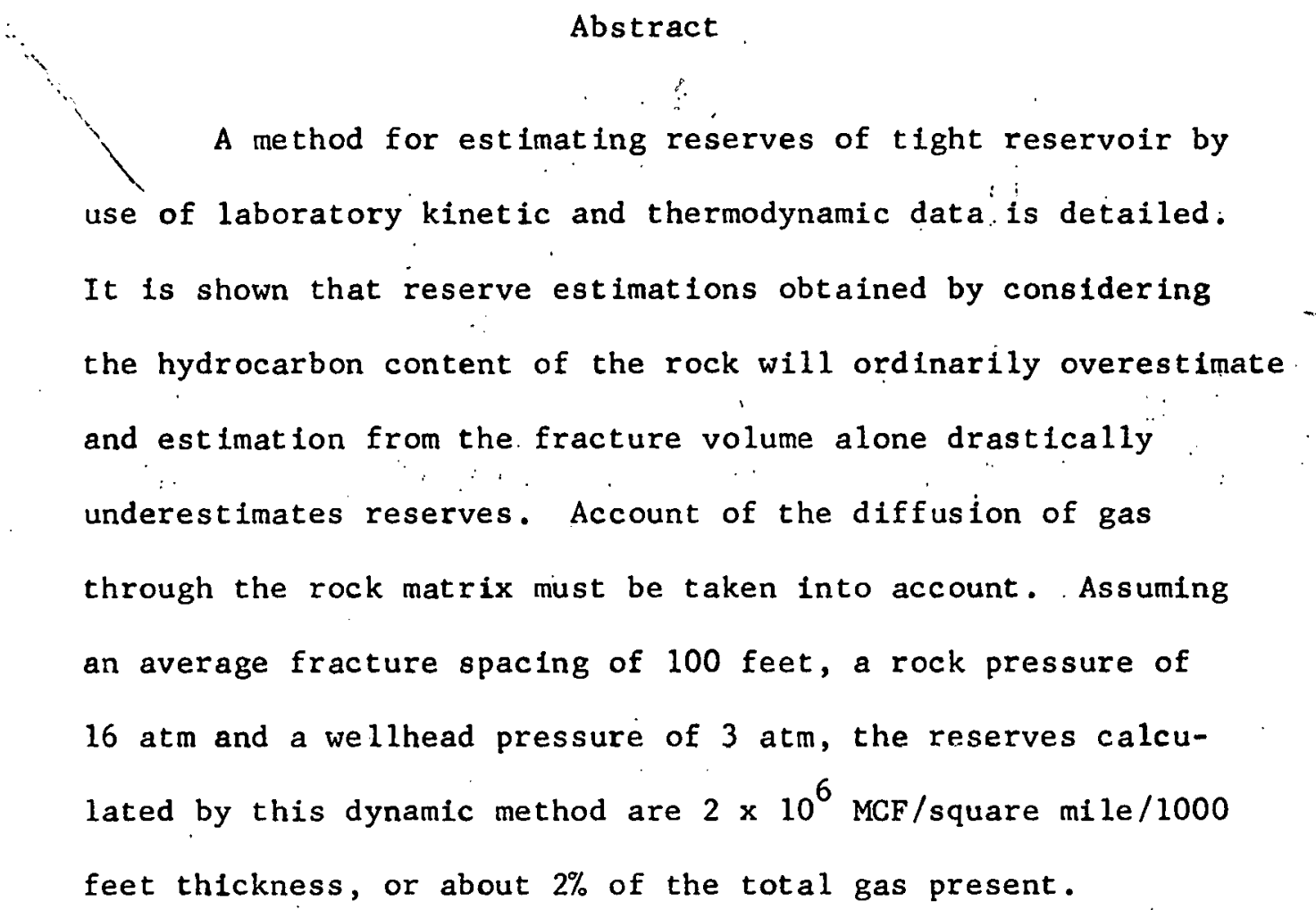


The determination of usable reserves in tight formations such as Devonian shale is not a simple task because specific account must be taken of the relatively complex processes resulting in production and the several parameters involved in this process must be estimated. The most direct approach would be to estimate the volume of rock drained by an (average) well and to estimate the total production to be expected from a test well on the basis of its initial productivity. In this approach the reserves associated with a reservolr would be calculated from the estimation of the volume of rock drained from a given well and the estimate of total well productivity. However, the total production of a given well does not seem to be a definable quantity because the productivity vs. time curves are (typically) very flat after the first few years and thus how much gas one eventually obtains is strongly dependent upon the length of time considered. Since the volume of gas associated with a given quantity of rock is a well defined (definite) quantity, one is led inexorably to the conclusion that the volume of rock drained by a given well also cannot be well defined. If neither the total production of a well nor. the volume of rock that is drained are definable properties, it would appear that the behavior of a single well is not a good predictor of the reserves that can be associated with a reservoir.

Clearly the total amount of gas in the reservoir can be taken as an estimate of the potential reserves and this can be estimated from samples of the rock either by direct outgassing studies or by isotherm measurements. We estimate that Devonian shales that we have studied contain approximately $8.2 \times 10^{7}$ MCF of gas per square mile per thousand feet thickness of shale by virtue of our averaged isotherm measurements $\left(3 \times 10^{-4} \mathrm{v} / \mathrm{v} /\right.$ torr $)$ and assuming the average rock pressure to be 16 atm with draw down to 3 atm possible. (1) Estimates of reserves obtained in this manner are, however, upper bounds that may be grossly optimistic. Not all the gas present in the rock will be produced during the practical life uf the wells and the question of the percentage of the total gas that can be produced 
In practice becomes an important one. One obvious contributor to unproducible gas is enclosed voids within rock with no connection to the open pore system. A more important contributor, however, is gas that lies too far from a macroscopic fracture that can serve as a conduit to the well bore. The diffusion constant of methane in shale is approximately $5 \times 10^{-7} \mathrm{~cm}^{2} / \mathrm{sec}$ (average) and this corresponds roughly to an average migration distance for methane through the rock of only 2 feet in 100 years. It thus seems clear that gas in shale that is more than a few feet away from a natural fracture is to all intents unproducible except insofar às the natural fracture system may be augmented by artifical fractures. Wherèas useful reserves of a tight reservoir are overestimated by simply calculating the total amount of gas in the rock, it has become apparent that the gas contained in the open volume of the fractures contributes only negligibly to the total gas produced. This is because this gas is smallin comparison to the gas that diffuses into the fracture through its faces. The amount of gas, " $\mathrm{M}_{0}$, produced from the volume associated with a fracture is

$$
\text { 1) } \mathrm{M}_{0}=\frac{\mathrm{A}}{2} \mathrm{X}\left(\mathrm{P}_{\mathrm{S}}-\mathrm{P}_{0}\right)
$$

where $X$ is the fracture width, $A$ is its area and $P_{S}$ is the initial rock pressure in atmospheres and $P_{0}$ is the final pressure at the end of production. (The $A / 2$ appears because of the two faces of the fracture.) However, the amount of gas diffusing through the side of the fracture is (2)

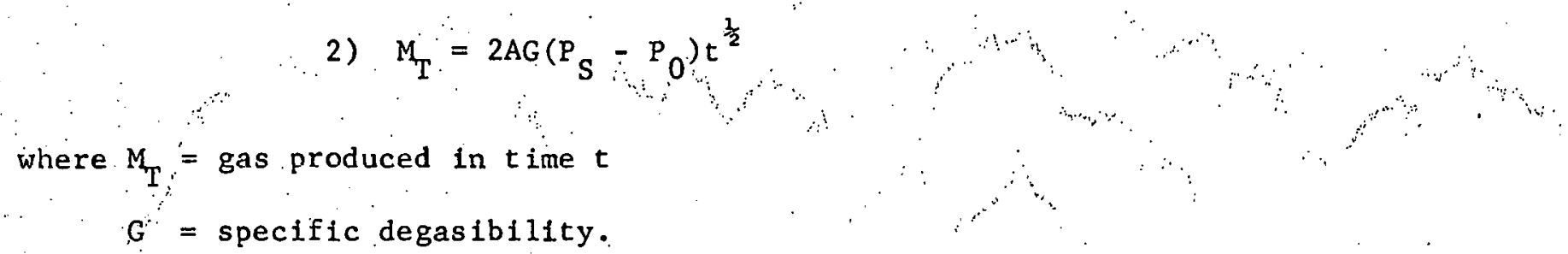

As a well begins to produce gas is obtained by simple PV expansion from the fractures. This is followed subsequently by production due to difusion throughk" the surfaces. The time that transition from the first process to the second 


.


occurs can be defined as that when $M_{T}=M_{0}$ from which 1) and 2) yield

$$
\text { 3) } t_{0}=\left(\frac{x}{4 G}\right)^{2}
$$

Both typical and extreme values of $G$ give $t_{0}$ 's that are very small in comparison to the lifetime of a well. Using an average value of degasibility of $2 \times 10^{-7}$ $\mathrm{cm}^{3} / \mathrm{cm}^{2} /$ torr $/ \mathrm{sec}^{\frac{1}{2}}$ and a mean fracture width of $.01 \mathrm{~cm}, t_{0}$ equals 4 minutes. For a worst case of $\mathrm{G}=2 \times 10^{-8}$ and $\mathrm{X}=.1 \mathrm{~cm}, \mathrm{t}_{0}$ equals 32 days. This means that the time associated with draining of the fractures is ordinarily of the order of minutes after which the source of the gas is the rock matrix., Because the time scale for production is years it would appear that although fractures are vitally Important as conduits to the well bore. they contain only negligible amounts of gas in comparison to that obtained by diffusion through the matrix.

As has been discussed ${ }^{(2)}$ an accurate model to describe the productivity of a shale well must be at least pseudo two step if the model is expected to be complete in the sense of predicting optimal well spacing, etc. Specific account must be taken of both the permeability through the fractures as well as diffusion through the rock to the fractures and neither step is rate limiting for all cases. Some progress has been made with such a two pseudo step theory ${ }^{(3)}$ but more fundamental theoretical work remains in order to develop solutions that are easily applicable. However, certain questions are readily dealt with by a pseudo one step model and in our present opinion the question of the usable reserves associated with a tight reservoir is among them.

Whereas the effect of the diffusion of gas through the rock is a constant factor that always must be dealt with for any calculation involving production or reserves in tight formation, the effect of the permeability of the fractures depends upon the fracture length, width, proppants used during stimulation and so forth. Given this state of affairs it seems that one measure of useful reserves is simply the total amount of gas that can diffuse into the natural fracture system from the matrix in some standard time such as 100 years for some assumed uniform 
pressure drop in the fracture system (due to well drilling). In this case the more general 2 step model reduces to a pseudo one step model which involves only the effects of matrix diffusion. The solution to the differential equations for this case are well known and can be expressed as ${ }^{(4)}$

$$
M_{T}=2 V_{s} G\left(P_{2}-P_{0}\right) t^{\frac{1}{2}}
$$

where $V$ is the volume of rock involved and $s$ is the fracture frequency expressed in area/volume. (Vs is the total natural fracture area of the reservoir.)

If one associates $M_{T}$ with the total producible gas in a volume square mile by 1000 feet thick

$$
M_{T}=2.0 \times 10^{8} \times M C F
$$

where $s$ is to be expressed in sq. ft. area/cu. ft. For plane fractures $s$ may be taken as the reciprocal of the average spacing." The equation applies only for low values of $s$ corresponding to the fracture spacing being larger than the depletion zones extending out from the fracture faces $(2 \times 2 \mathrm{ft}$ for the average case after 100 years of production). For its region of validity $M_{T}$ calculated in this way will be less than $\mathrm{M}_{\mathrm{T}}$ estimated from the total methane content and represents a more reasonable upper bound to the amount of gas obtainable from a reservoir.

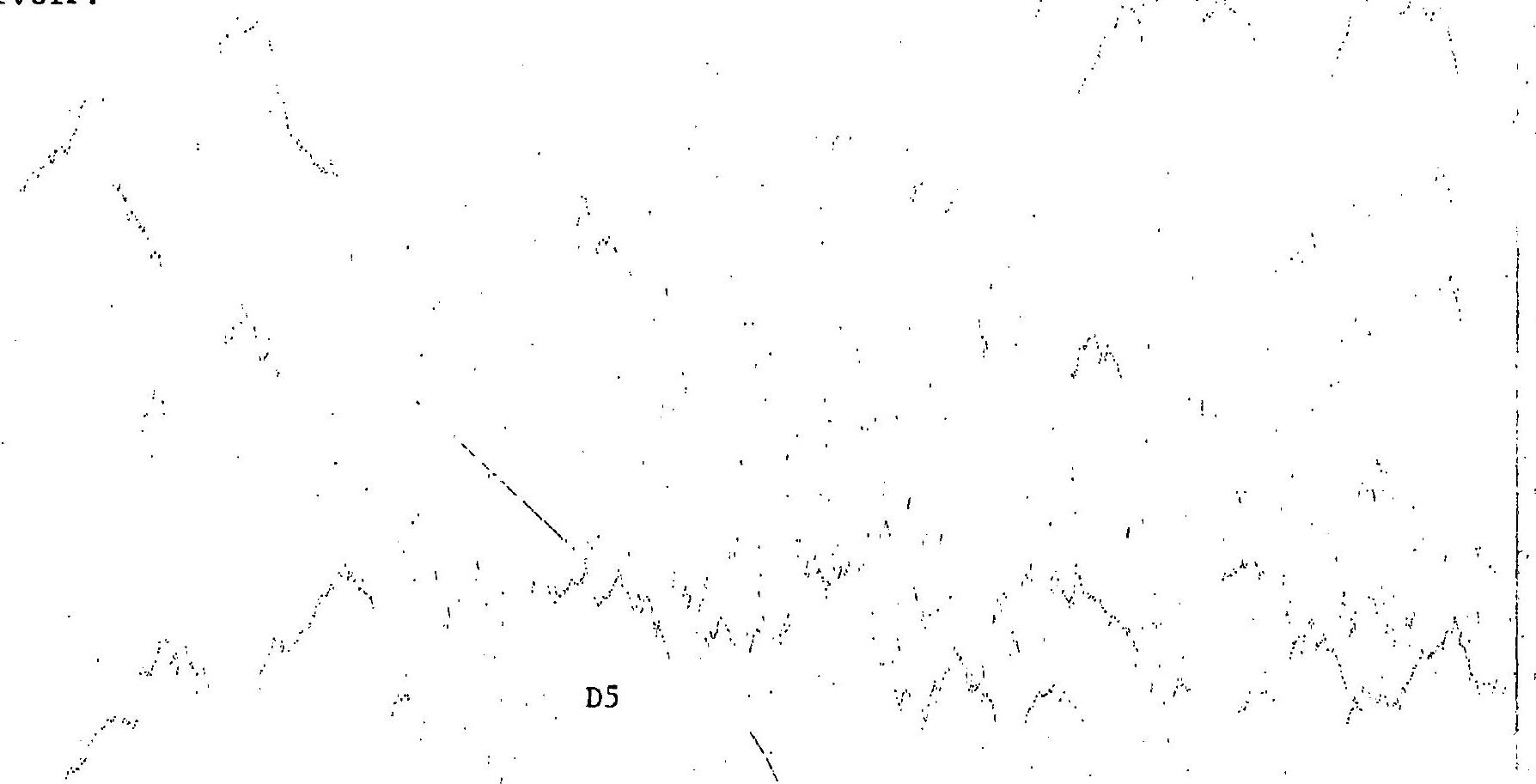




\section{$\underline{\text { References }}$}

1. The following progrese reports for Contract No. EY-76-S-05-5197 on "Study of Hydrocarbon Shale Interaction" contain most of our laboratory kinetic and thermodynamic measurements: Annual Report 非1, OR0-5197-6; Progress Report 非9, OR0-5197-9; Progress Report 非10, OR0-5197-10.

2. "The Implications of Specific Degasibility for Models of Gas Production from Shale," Paul D. Schettler, Proceedings of the Second Annual Eastern Gas Shales Symposium, Morgantown, West Virginia, October 1978.

3. "Gas Production in Wells: Constrictive and Geometric Effects," Paul D. Schettler, with Progress Report 非1, ORO-5197-1 of Contract No. EY-76-S-05-5197.

4. "The Concept of Specific Degasibility and Its Application to the Gas Bearing Tight Formations as Represented by the Devonian Shales of Appalachia,". Paul D. Schettler, Proceedings of the Fourth Annual DOE Symposium on Enhanced 011 \& Gas Recovery, VII, G8/1. 
ORO-5197-10

Appendix E

PROGRESS REPORT

INFRARED AND X-RAY STUDIES

William E. Russey

Juniata College

Huntingdon, Pennsylvania 16652 

PROGRESS REPORT

INFRARED AND X-RAY STUDIES

Preliminary studies were intiated with the aim of investigating possible correlations between gas interaction parameters and infrared spectral characteristics of shale.samples. Progress to date has been limited to developing appropriate sampling techniques, acquiring a familiarity with the techniques generally, assessing the probable importance of various spectral regions, and selecting a series of samples for further analysis, these being chosen to reflect both typical and extreme values of diffusion and isotherm parameters. The long wavelength $(\mathrm{KBr})$ region of the infrared spectrum appears to show considerable promise in providing spectral diversity, and initial efforts will be directed toward pursuing this prospect.

It is the goal of this phase of the project to ftrd rapid, routine analysis techniques which will allow a degree of prediction of gas interaction parameters, and it is hoped that the results of infrared analysis can be combined in a meaningful way with X-ray diffraction measurements to yield additional insight into the causes of variation from sample to sample. 